\title{
FIRST-YEAR SPECTROSCOPY FOR THE SLOAN DIGITAL SKY SURVEY-II SUPERNOVA SURVEY
}

Chen Zheng $^{1}$, Roger W. Romani ${ }^{1}$, Masao Sako $^{2}$, John Marriner $^{3}$, Bruce Bassett ${ }^{4,5}$, Andrew Becker $^{6}$, Changsu Choi $^{7}$, David Cinabro $^{8}$, Fritz DeJongh ${ }^{3}$, Darren L. Depoy ${ }^{9}$, Ben Dilday ${ }^{10,11}$, Mamoru Doi ${ }^{12}$, Joshua A. Frieman ${ }^{3,11,13}$, Peter M. Garnavich ${ }^{14}$, Craig J. Hogan ${ }^{6}$, Jon Holtzman ${ }^{15}$, Myungshin Im ${ }^{7}$, Saurabh Jha $^{1}$, Richard Kessler $^{11,16}$, Kohki Konishi ${ }^{17}$, Hubert Lampeitl ${ }^{18}$, Jennifer L. Marshall ${ }^{9}$, David McGinnis ${ }^{3}$, Gajus Miknaitis ${ }^{3}$, Robert C. Nichol ${ }^{19}$, Jose Luis Prieto ${ }^{9}$, Adam G. Riess ${ }^{18,20}$, Michael W. Richmond ${ }^{21}$, Donald P. Schneider ${ }^{22}$, Mathew Smith ${ }^{19}$,

NaOhiro Takanashi ${ }^{12}$, Kouichi Tokita ${ }^{12}$, Kurt van der Heyden ${ }^{5}$, Naoki Yasuda ${ }^{17}$, Roberto J. Assef ${ }^{9}$, John Barentine ${ }^{23,24}$, Ralf Bender ${ }^{25,26}$, Roger D. Blandford ${ }^{1}$, Malcolm Bremer ${ }^{27}$, Howard Brewington ${ }^{24}$, Chris A. Collins ${ }^{28}$, Arlin Crotts ${ }^{29}$, Jack Dembicky $^{24}$, Jason Eastman $^{9}$, Alastair Edge $^{30}$, Ed Elson $^{4,5}$, Michael E. Eyler ${ }^{31}$, Alexei V. Filippenko ${ }^{32}$, Ryan J. Foler ${ }^{32}$, Stephan Frank ${ }^{9}$, Ariel Goobar ${ }^{33}$, Michael HarvaneK ${ }^{24,34}$, Ulrich Hopp $^{25,26}$, Yutaka Ihara ${ }^{12}$, Steven Kahn $^{1}$, William Ketzeback ${ }^{24}$, Scott J. Kleinman ${ }^{24,35}$, Wolfram Kollatschny ${ }^{36}$, Jurek Krzesiński ${ }^{24,37}$, Giorgos Leloudas ${ }^{38}$, Daniel C. Long ${ }^{24}$, John LuCEY ${ }^{30}$, Elena Malanushenko ${ }^{24}$, Viktor Malanushenko ${ }^{24}$, Russet J. McMillan ${ }^{24}$, Christopher W. Morgan ${ }^{9,31}$, Tomoki Morokuma ${ }^{12,39}$, Atsuko Nitta ${ }^{24,40}$, Linda Ostman ${ }^{33}$, Kaike Pan $^{24}$, A. Kathy Romer ${ }^{41}$, Gabrelle Saurage $^{24}$, Katie Schlesinger ${ }^{9}$, Stephanie A. Snedden ${ }^{24}$, Jesper Sollerman ${ }^{38,42}$, MaXimilian Stritzinger $^{38}$, Linda C. Watson $^{9}$, Shannon Watters ${ }^{24}$, J. Craig WheEler ${ }^{23}$, AND DONAld YorK ${ }^{13,16}$

${ }^{1}$ Kavli Institute for Particle Astrophysics and Cosmology, Stanford University, Stanford, CA 94305-4060, USA

${ }^{2}$ Department of Physics and Astronomy, University of Pennsylvania, 209 South 33rd Street, Philadelphia, PA 19104, USA

${ }^{3}$ Center for Particle Astrophysics, Fermi National Accelerator Laboratory, P.O. Box 500, Batavia, IL 60510, USA

${ }^{4}$ Department of Mathematics and Applied Mathematics, University of Cape Town, Rondebosch 7701, South Africa ${ }^{5}$ South African Astronomical Observatory, P.O. Box 9, Observatory 7935, South Africa

${ }^{6}$ Department of Astronomy, University of Washington, Box 351580, Seattle, WA 98195, USA

${ }^{7}$ Department of Physics \& Astronomy, FPRD, Seoul National University, Seoul, South Korea ${ }^{8}$ Department of Physics, Wayne State University, Detroit, MI 48202, USA

${ }^{9}$ Department of Astronomy, Ohio State University, 140 West 18th Avenue, Columbus, OH 43210-1173, USA

${ }^{10}$ Department of Physics, University of Chicago, Chicago, IL 60637, USA

${ }^{11}$ Kavli Institute for Cosmological Physics, The University of Chicago, 5640 South Ellis Avenue Chicago, IL 60637, USA

${ }^{12}$ Institute of Astronomy, Graduate School of Science, University of Tokyo 2-21-1, Osawa, Mitaka, Tokyo 181-0015, Japan

${ }^{13}$ Department of Astronomy and Astrophysics, The University of Chicago, 5640 South Ellis Avenue, Chicago, IL 60637, USA ${ }^{14}$ University of Notre Dame, 225 Nieuwland Science, Notre Dame, IN 46556-5670, USA

${ }^{15}$ Department of Astronomy, MSC 4500, New Mexico State University, P.O. Box 30001, Las Cruces, NM 88003, USA

${ }^{16}$ Enrico Fermi Institute, University of Chicago, 5640 South Ellis Avenue, Chicago, IL 60637, USA

${ }^{17}$ Institute for Cosmic Ray Research, University of Tokyo, 5-1-5, Kashiwanoha, Kashiwa, Chiba, 277-8582, Japan

${ }^{18}$ Space Telescope Science Institute, 3700 San Martin Drive, Baltimore, MD 21218, USA

${ }^{19}$ Institute of Cosmology and Gravitation, Mercantile House, Hampshire Terrace, University of Portsmouth, Portsmouth PO1 2EG, UK

${ }^{20}$ Department of Physics and Astronomy, Johns Hopkins University, 3400 North Charles Street, Baltimore, MD 21218, USA

${ }^{21}$ Physics Department, Rochester Institute of Technology, 85 Lomb Memorial Drive, Rochester, NY 14623-5603, USA

${ }^{22}$ Department of Astronomy and Astrophysics, The Pennsylvania State University, 525 Davey Laboratory, University Park, PA 16802, USA

${ }^{23}$ Department of Astronomy, McDonald Observatory, University of Texas, Austin, TX 78712, USA

${ }^{24}$ Apache Point Observatory, P.O. Box 59, Sunspot, NM 88349, USA

${ }^{25}$ Universitaets-Sternwarte Munich, 1 Scheinerstr, Munich, D-81679, Germany

${ }^{26}$ Max Planck Institute for Extraterrestrial Physics, D-85748, Garching, Munich, Germany

${ }^{27}$ H. H. Wills Physics Laboratory, University of Bristol, Bristol, BS8 1TL, UK

${ }^{28}$ Astrophysics Research Institute, Liverpool John Moores University, Birkenhead CH41 1LD, UK

${ }_{29}$ Department of Astronomy, Columbia University, New York, NY 10027, USA

${ }^{30}$ Department of Physics, University of Durham, South Road, Durham, DH1 3LE, UK

${ }^{31}$ Department of Physics, United States Naval Academy, 572C Holloway Road, Annapolis, MD 21402, USA

${ }^{32}$ Department of Astronomy, University of California, Berkeley, CA 94720-3411, USA

${ }^{33}$ Physics Department, Stockholm University, AlbaNova University Center, 106 91 Stockholm, Sweden

${ }^{34}$ Lowell Observatory, 1400 Mars Hill Rd., Flagstaff, AZ 86001, USA

${ }^{35}$ Subaru Telescope, 650 N. A'Ohoku Place, Hilo, HI 96720, USA

${ }^{36}$ Institut für Astrophysik, Universität Göttingen, Friedrich-Hund-Platz 1, D-37077 Göttingen, Germany

${ }^{37}$ Obserwatorium Astronomiczne na Suhorze, Akademia Pedagogicazna w Krakowie, ulica Podchorążych 2, PL-30-084 Kraków, Poland

${ }^{38}$ Dark Cosmology Centre, Niels Bohr Institute, University of Copenhagen, DK-2100, Denmark

${ }^{39}$ National Astronomical Observatory of Japan, 2-21-1, Osawa, Mitaka, Tokyo 181-8588, Japan

${ }^{40}$ Gemini Observatory, 670 North A'ohoku Place, Hilo, HI 96720, USA

${ }^{41}$ Astronomy Center, University of Sussex, Falmer, Brighton BN1 9QJ, UK

42 Astronomy Department, Stockholm University, AlbaNova University Center, 10691 Stockholm, Sweden Received 2007 October 28; accepted 2008 February 18; published 2008 April 7

\section{ABSTRACT}

This paper presents spectroscopy of supernovae (SNe) discovered in the first season of the Sloan Digital Sky Survey-II SN Survey. This program searches for and measures multi-band light curves of SNe in the redshift range $z=0.05-0.4$, complementing existing surveys at lower and higher redshifts. Our goal is to better characterize the SN population, with a particular focus on SNe Ia, improving their utility as cosmological distance indicators and as probes of dark energy. Our SN spectroscopy program features rapid-response observations using telescopes of a range of apertures, and provides confirmation of the SN and host-galaxy types as well as precise redshifts. 
We describe here the target identification and prioritization, data reduction, redshift measurement, and classification of $129 \mathrm{SNe} \mathrm{Ia}, 16$ spectroscopically probable SNe Ia, $7 \mathrm{SNe}$ Ib/c, and $11 \mathrm{SNe}$ II from the first season. We also describe our efforts to measure and remove the substantial host-galaxy contamination existing in the majority of our SN spectra.

Key words: cosmology: observations - methods: data analysis - supernovae: general - surveys - techniques: spectroscopic

Online-only material: color figures

\section{INTRODUCTION}

During the last few decades, studies of Type Ia supernovae (SNe Ia) have made significant contributions to our understanding of cosmology. In particular, SN Ia samples from low redshifts $(z \lesssim 0.1)$ have helped to constrain the Hubble constant (Hamuy et al. 1996; Jha et al. 1999, 2007). Their comparison with SN Ia samples from high redshifts $(z \gtrsim 0.4)$ has led to evidence for an accelerating universe (Riess et al. 1998; Perlmutter et al. 1999; see Filippenko 2005b for a review). The key is that these $\mathrm{SNe}$ prove to be remarkably homogeneous "standard candles" after correcting for the empirical luminosity versus decline-rate relation (e.g., Phillips 1993). However, surveys covering large areas with intermediate depth have proved difficult to perform, leading to a "redshift desert" $(0.1 \lesssim z \lesssim 0.4$; Riess et al. 2004) in the SN Ia Hubble diagram. Further, merging data from multiple surveys raises questions of systematics control; high-precision cross calibration is required to differentiate between cosmological models. To further calibrate the $\mathrm{SN}$ luminosities, to probe for possible redshift evolution, and to study the empirical properties of the "dark energy" that has been invoked to account for the acceleration of the universe, it is critical to fill in this redshift desert with well-studied data and to connect the low- $z$ and high- $z$ populations.

The SN survey of the Sloan Digital Sky Survey-II (SDSS-II), comprising three three-month campaigns (2005 September through 2007 November), was launched to discover $\mathrm{SNe}$ Ia and acquired photometric and spectroscopic observations in this sparsely sampled intermediate-redshift interval of $z=0.05-0.4$ (Frieman et al. 2007). The large (300 deg ${ }^{2}$, moderately deep $(r \approx 22.5 \mathrm{mag})$ survey provides a significant volume for untargeted discovery of these intermediate-redshift $\mathrm{SNe}$, thus complementing ongoing low- $z$ surveys and followup programs (e.g., Lick Observatory SN Search (LOSS), ${ }^{43}$ Li et al. 2000; Filippenko et al. 2001; Filippenko 2005a; Carnegie SN Program (CSP) ${ }^{44}$ Hamuy et al. 2006; Nearby SN Factory (SNFactory), ${ }^{45}$ Copin et al. 2006; the Center for Astrophysics follow-up effort (CfA) SN Group, ${ }^{46}$ Riess et al. 1995, 1999; Riess 1996; Jha et al. 2006, 2007). The survey is sufficiently deep to overlap with the high- $z$ samples (e.g., Canada-France-Hawaii Telescope SN Legacy Survey (SNLS) ${ }^{47}$ Astier et al. 2006; Equation of State: SNe trace Cosmic Expansion (ESSENCE), ${ }^{48}$ Miknaitis et al. 2007; WoodVasey et al. 2007) and help to explore the kinematics of the expanding universe during the interval when most models of dark energy anticipate maximum departure from a simple, flat, $\Lambda$-Cold Dark Matter (LCDM) cosmology. The uniformity of

43 http://astro.berkeley.edu/ bait/kait.html

44 http://csp1.lco.cl/ cspuser1/PUB/CSP.html.

$45 \mathrm{http} / / /$ snfactory.lbl.gov/.

46 http://www.cfa.harvard.edu/cfa/oir/Research/supernova/SNgroup.html.

47 http://www.cfht.hawaii.edu/SNLS

48 http://www.ctio.noao.edu/essence. the SDSS photometric calibration system provides precise measurements of SN light curves in five filters (ugriz; Fukugita et al. 1996). The large survey volume also facilitates discovery of rare SN types, sampling the full extent of the SN Ia population, as well as allowing searches for $\mathrm{SNe} \mathrm{Ib} / \mathrm{c}, \mathrm{SNe}$ II, hypernovae, and other peculiar denizens of the astronomical zoo.

This paper describes our spectroscopic follow-up techniques and presents results from spectroscopic observations of $\mathrm{SNe}$ discovered in the first season of the SDSS-II SN Survey. During the Fall 2005 campaign, we repeatedly scanned SDSS stripe 82, with alternate observations of the northern and southern strips, maintaining full coverage of the $2.5^{\circ}$ wide stripe on a cadence of $\sim 2 \mathrm{~d}$. SN candidates were identified by rapid on-mountain processing in the $g, r$, and $i$ filters. Targets that passed a variety of quality control cuts were then inspected by humans and prioritized for spectroscopic follow-up observations (Sako et al. 2007). The primary spectroscopic facilities used to complement the imaging survey were the $2.4 \mathrm{~m}$ Hiltner telescope at MDM, the $3.5 \mathrm{~m}$ Astronomy Research Consortium (ARC) telescope at Apache Point Observatory (APO), and the queue-scheduled 9.2 m Hobby-Eberly Telescope (HET) at McDonald Observatory. Individual observing campaigns were also conducted with the $4.2 \mathrm{~m}$ William Herschel Telescope (WHT), the $8.2 \mathrm{~m} \mathrm{Sub-}$ aru, and the $10 \mathrm{~m}$ Keck I telescope. A total of 259 spectra were obtained during the first season, yielding 129 spectroscopically confirmed SNe Ia with a wide range of epochs $(-12 \mathrm{~d}$ to $54 \mathrm{~d}$ relative to $B$-band maximum light), 16 spectroscopically probable SNe Ia, and a handful of other SN types, including peculiar $\mathrm{SNe}$ Ia and broad-lined SN Ib/c "hypernovae." Several objects were observed at multiple epochs.

In this paper, we present our spectroscopic data reduction and analysis methods. We briefly summarize the candidate identification and prioritization algorithms (Section 2), and we describe the spectroscopic observations at several follow-up telescopes as well as the basic data reduction (Section 3). In Section 4, we describe the procedures used for SN classification and redshift determination, including efforts to measure and remove spectral contamination by the host-galaxy light. Section 5 summarizes the basic results, describing a few of the peculiar spectra. A complete analysis of the spectral features in the SN Ia population and extension of the analysis to Seasons 2 and 3 are deferred to later publications. However, in Section 6, we note potential results from the analysis of the full spectroscopy data and summarize our present conclusions.

\section{SELECTION OF SUPERNOVA CANDIDATES FOR SPECTROSCOPY}

The SDSS-II SN Program uses a CCD camera (Gunn et al. 1998) mounted on the APO $2.5 \mathrm{~m}$ telescope (Gunn et al. 2006) to obtain repeated scans of a $300 \mathrm{deg}^{2}$ region aligned along the 
celestial equator in the Southern Galactic Hemisphere. Images in this $2.5^{\circ} \times 120^{\circ}$ area (SDSS Stripe 82) are obtained almost simultaneously using five filters (ugriz; Fukugita et al. 1996). The images have sufficient sensitivity to discover SNe Ia out to $z \approx 0.4$, while providing high-quality light curves (J. Holtzman et al. 2007, in preparation) for a large fraction of the $\mathrm{SNe}$ on an accurately calibrated photometric system $(\sim 1 \%$; Ivezic et al. 2007). Technical summaries of the SDSS and the data products can be found in York et al. (2000), Adelman-McCarthy et al. (2007), and references therein. An overview of the SDSS-II SN program is presented by Frieman et al. (2007).

With the large data volume produced for each clear night, it was necessary to flag SN candidates using a dedicated computer cluster at APO. Co-added template images from previous years were registered and convolved to match the search images in the $g, r$, and $i$ bands. Statistically significant variable sources appearing in two or more filters were flagged. Within $24 \mathrm{~h}$, thousands of new detections from each night of observation were transferred to Fermilab and entered into the SN database. These detections (or "objects") were visually inspected to identify possible SN candidates. Host-galaxy properties (morphology, color, and photometric redshift) were assembled along with estimates for the SN photometric redshift, extinction, date of maximum light, photometric type, and stretch factor, as obtained from the preliminary photometry. For all active candidates, these parameters were updated daily. Visual grading of the candidates produced a cleaner SN set. Efficiencies for selecting true $\mathrm{SNe}$ were actively monitored with a large number of "fake" $\mathrm{SNe}$ added to the data stream. Details of the candidate identification and characterization are described by Sako et al. (2007).

With such a large number of candidate $\mathrm{SNe}$ and limited spectroscopic resources, only the "best" objects were subject to follow-up spectroscopic observations. In particular, during the first season, we focused (with a few exceptions) on objects for which a classification of SN Ia was strongly preferred by the initial photometry. We also selected for large SN-galaxy separation, minimal photometric evidence for dust extinction, and interesting $\mathrm{SNe}$ (those with lowest or highest redshift, underluminous hosts, and peculiar light curves). This approach certainly biases the distribution of SN properties, except for $z \lesssim 0.12$, where we attempted to make the data as complete as possible (Dilday et al. 2008). These "best" SNe were assigned to the various telescopes: APO, MDM, and WHT for the brighter objects with lower-estimated redshifts, or HET and Subaru for the fainter distant objects. Significant efforts were made to avoid duplicate observations and to maximize the observation efficiency of each telescope. Only a modest fraction $(\sim 25 \%)$ of the selected candidates were not observed within 20 restframe days of SN maximum or yielded noisy spectra; hostgalaxy spectra and redshifts are being measured for a sample of those objects missed due to poor weather or oversubscribed spectroscopic resources.

\section{OBSERVATIONS AND REDUCTIONS}

During fall 2005, three telescopes were routinely available for SDSS-II SN spectroscopy: MDM $2.4 \mathrm{~m}$ (49 shared nights), ARC $3.5 \mathrm{~m}$ (31 half-nights), and HET $9.2 \mathrm{~m}$ (64.5 h of queue time). Several additional dedicated campaigns helped greatly, with six nights on the $4.2 \mathrm{~m}$ WHT, six shared nights on Subaru, and one target-of-opportunity night on Keck. A handful of additional spectra were obtained at other facilities based on the SDSSII SN candidate announcements. In total, 259 useful spectra were obtained which yielded 129 spectroscopically confirmed

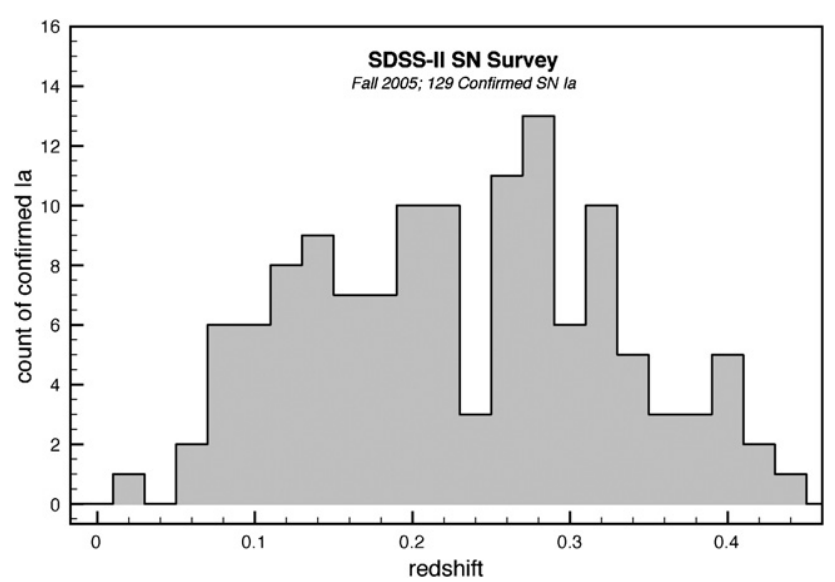

Figure 1. The redshift distribution of 129 spectroscopically confirmed SNe Ia in 2005 .

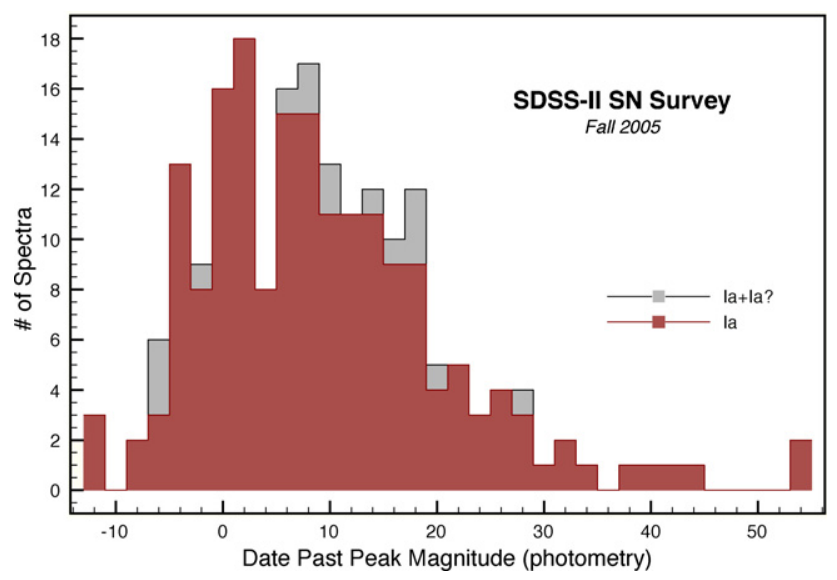

Figure 2. Distribution of first spectroscopy epoch, relative to $B$-band maximum light, for spectroscopically confirmed and probable SNe Ia; most spectra were obtained before $+20 \mathrm{~d}$.

(A color version of this figure is available in the online journal)

SNe Ia, 16 probable $\mathrm{SNe}$ Ia, $7 \mathrm{SNe} \mathrm{Ib} / \mathrm{c}$, and $11 \mathrm{SNe}$ II. Figure 1 shows the redshift distribution of the confirmed SNe Ia. The distribution is approximately flat for $0.1<z<0.35$ with a deficit at $z \approx 0.2$. This deficit is almost purely a result of bias in our follow-up selection, with bright low- $z$ sources assigned to the smaller telescopes and distant sources targeted at the $\geqslant 8$ $\mathrm{m}$ facilities.

Most SNe had spectroscopy within $20 \mathrm{~d}$ of photometric maximum (Figure 2). Multi-epoch follow-up spectroscopy was carried out for the peculiar Type Ia SN 2005js (two epochs; similar to SN 1991bg-e.g., Filippenko et al. 1992b), the underluminous, low-expansion velocity Type Ia SN 2005hk (ten epochs; see also Chornock et al. 2006; Phillips et al. 2007), and a peculiar event, SN 2005gj (23 epochs; see also Aldering et al. 2006; Prieto et al. 2007), which had broad SN Ia-like features with superimposed hydrogen emission lines (similar to SN 2002ic, Hamuy et al. 2003). Three broad-lined Type Ic "hypernovae" (similar to SN 1998bw and SN 2002ap; e.g., Stathakis et al. 2000; Foley et al. 2003) were observed as well: SN 2005fk, SN 2005kr, and SN 2005ks, selected as Type Ic "hypernovae" based on their light curves, each had a SN 2002aplike spectrum. At least some "hypernovae" are believed to be associated with gamma-ray bursts (e.g., Stanek et al. 2003; Matheson et al. 2003). 
Here we summarize the observing configurations for the main facilities. The individual targeted $\mathrm{SN}$ positions and dates of observations are listed in Table 1.

\subsection{Telescopes \\ 3.1.1. MDM $2.4 \mathrm{~m}$}

The MDM $2.4 \mathrm{~m}$ telescope was used for both imaging and spectroscopy of the SN candidates. With a large number of nights and an efficient spectrograph, this facility made important contributions despite its modest aperture. Spectra were taken with the Boller \& Chivens CCD Spectrograph (CCDS), with a $1501 \mathrm{~mm}^{-1}$ grating (4700 ̊ blaze) feeding a $1200 \times 800$ Loral CCD. With a $2^{\prime \prime}$ slit we obtained a resolution of $15 \AA$ covering 3800-7300 A. Observations with the fixed north/south slit were taken within $1 \mathrm{~h}$ of transit, whenever possible, to minimize refractive slit losses (Filippenko 1982). Typically the exposure was $3 \times 900 \mathrm{~s}$. A total of 38 spectra were obtained confirming $16 \mathrm{SNe} \mathrm{Ia}$, two $\mathrm{SNe} \mathrm{Ib/c,} \mathrm{and} \mathrm{four} \mathrm{host} \mathrm{galaxies,} \mathrm{with} \mathrm{a}$ redshift distribution centered at $z \approx 0.08$. This telescope was used extensively to perform multi-epoch spectroscopy of bright, nearby events.

\subsubsection{ARC $3.5 \mathrm{~m}$}

The 54 ARC spectra were obtained with the Dual Imaging Spectrograph (DIS), which has dual (red/blue) cameras with Marconi $2048 \times 1024$ pixel back-illuminated chips. The blue spectra used a $3001 \mathrm{~mm}^{-1}$ grating giving $2.43 \AA$ pixel $^{-1}$ centered at $4224 \AA$; the red camera employed a $3001 \mathrm{~mm}^{-1}$ grating for $2.26 \AA$ pixel $^{-1}$ dispersion, centered at $7500 \AA$. With a slit width of $\sim 1.5^{\prime \prime}$, the effective resolution was $\sim 8-9 \AA$. For most observations with a visible host, the slit was aligned with the SN and galaxy core. Typical exposures were 300-900 s and 3-5 exposures were combined for the final spectra. The observations yielded $38 \mathrm{SNe}$ (29 SNe Ia, three probable $\mathrm{SNe}$ Ia, five $\mathrm{SNe}$ II, and one $\mathrm{SN} \mathrm{Ib}$ ), with a median redshift of $\sim 0.11$.

\subsubsection{HET $9.2 \mathrm{~m}$}

The HET was used to observe SN candidates with higher photo- $z$. Exposures were made with the Marcario LowResolution Spectrograph (LRS; Hill et al. 1998), employing a Ford Aerospace $3072 \times 1024$ pixel chip and the $3001 \mathrm{~mm}^{-1} \mathrm{G} 1$ grism for a dispersion of $5 \AA$ pixel $^{-1}$. With a slit width of $2^{\prime \prime}$, the effective resolution was $\sim 20 \AA$. Observations were made with the slit at the parallactic angle (Filippenko 1982) to avoid differential slit losses and improve (relative) spectrophotometry across the range 4067-10700 $\AA$. As the HET is queue scheduled (Shetrone et al. 2007), many SN spectra were obtained soon after discovery, and lower-priority observation time was spent acquiring late-time data for $\mathrm{SNe}$ with previous spectroscopic confirmation. We obtained 92 HET spectra over a period of 69 days, yielding $61 \mathrm{SNe}$ (45 SNe Ia, 10 probable SNe Ia, one $\mathrm{SN} \mathrm{Ib}$, two $\mathrm{SNe}$ II, and three $\mathrm{SNe} \mathrm{Ibc}$ ) with a redshift distribution centered at $z \approx 0.27$. A few multi-epoch observations and a few unclassifiable spectra completed the sample.

\subsubsection{WHT $4.2 \mathrm{~m}$}

Six nights of observing were obtained with the ISIS Double Beam Spectrograph. Spectra with a combined red/blue coverage of 3900-8900 A were obtained for 30 SN candidates. Typically
Table 1

SDSS-II SN Spectroscopic Follow-up Observations

\begin{tabular}{|c|c|c|c|c|c|}
\hline $\begin{array}{l}\text { IAU }^{\mathrm{a}} \\
\text { Name }\end{array}$ & $\mathrm{SNID}^{\mathrm{b}}$ & $\begin{array}{l}\text { R.A. } \\
\text { (J2000) }\end{array}$ & $\begin{array}{l}\text { Decl. } \\
(\text { J2000) }\end{array}$ & Telescope & $\begin{array}{l}\text { Obs. Date } \\
\text { (UT) }\end{array}$ \\
\hline 2005ed & 722 & 000249.36 & +004504.8 & APO & 2005 Sep 09 \\
\hline $2005 \mathrm{ef}$ & 739 & 005822.87 & +004044.6 & APO & 2005 Sep 09 \\
\hline 2005 ei & 744 & 215647.65 & +001903.0 & APO & 2005 Sep 08 \\
\hline 2005eg & 762 & 010208.49 & -005244.5 & APO & 2005 Sep 09 \\
\hline $2005 e x$ & 774 & 014151.24 & -005235.0 & APO & 2005 Sep 09 \\
\hline \multirow[t]{2}{*}{2005 ex } & 774 & 014151.24 & -005235.0 & MDM & 2005 Sep 15 \\
\hline & 779 & 014641.70 & -010114.1 & HET & 2005 Dec 29 \\
\hline $2005 \mathrm{ez}$ & 1032 & 030710.97 & +010710.4 & APO & 2005 Sep 25 \\
\hline $2005 f g$ & 1112 & 223604.20 & -002230.8 & HET & 2005 Sep 26 \\
\hline $2005 \mathrm{lb}$ & 1114 & 225450.06 & -001508.9 & $\mathrm{APO}$ & 2005 Nov 04 \\
\hline \multirow[t]{2}{*}{$2005 \mathrm{fc}$} & 1119 & 212139.25 & +005340.7 & Subaru & $2005 \mathrm{Sep} 27$ \\
\hline & 1166 & 003725.35 & +005823.8 & Subaru & 2005 Sep 28 \\
\hline $2005 \mathrm{ff}$ & 1241 & 223041.41 & -004635.7 & WHT & 2005 Sep 23 \\
\hline $2005 \mathrm{fd}$ & 1253 & 213511.76 & +000947.3 & WHT & 2005 Sep 24 \\
\hline $2005 \mathrm{fd}$ & 1253 & 213511.76 & +000947.3 & APO & 2005 Sep 24 \\
\hline $2005 \mathrm{fd}$ & 1253 & 213511.76 & +000947.3 & HET & 2005 Sep 26 \\
\hline $2005 \mathrm{fe}$ & 1316 & 221927.32 & +00 2939.9 & WHT & 2005 Sep 29 \\
\hline \multirow[t]{2}{*}{$2005 \mathrm{fh}$} & 1371 & 231729.71 & +002545.8 & APO & 2005 Sep 25 \\
\hline & 1461 & 013729.44 & +00 1235.0 & HET & 2005 Dec 31 \\
\hline \multirow[t]{3}{*}{ 2005lc } & 1472 & 030211.19 & -010959.4 & APO & 2005 Nov 06 \\
\hline & 1525 & 030849.93 & +000930.0 & HET & 2005 Dec 25 \\
\hline & 1545 & 005742.28 & -004042.3 & APO & 2005 Sep 09 \\
\hline \multirow[t]{6}{*}{$2005 \mathrm{fb}$} & 1580 & 030117.54 & -003838.6 & WHT & 2005 Sep 23 \\
\hline & 1595 & 212616.29 & -003314.6 & MDM & 2005 Sep 25 \\
\hline & 1632 & 213054.23 & -000802.2 & APO & 2005 Sep 28 \\
\hline & 1686 & 000859.60 & -001237.5 & Subaru & 2005 Sep 27 \\
\hline & 1688 & 212525.85 & +00 1928.3 & Subaru & 2005 Sep 28 \\
\hline & 1740 & 002137.06 & -005251.1 & HET & 2005 Dec 25 \\
\hline $2005 \mathrm{fj}$ & 1794 & 211120.85 & -002643.3 & WHT & 2005 Sep 24 \\
\hline $2005 \mathrm{hl}$ & 2000 & 205519.79 & +003234.7 & MDM & 2005 Oct 22 \\
\hline 2005 fo & 2017 & 215546.40 & +00 3536.7 & HET & 2005 Sep 22 \\
\hline $2005 \mathrm{fl}$ & 2030 & 204721.99 & -011511.9 & HET & 2005 Sep 27 \\
\hline $2005 \mathrm{fm}$ & 2031 & 204810.37 & $\begin{array}{llll}01 & 10 & 17.1\end{array}$ & WHT & 2005 Sep 23 \\
\hline \multirow[t]{2}{*}{$2005 \mathrm{fk}$} & 2053 & 211519.84 & -00 2258.6 & HET & 2005 Sep 27 \\
\hline & 2063 & 215923.79 & -010627.0 & HET & 2005 Sep 25 \\
\hline $2005 \mathrm{fn}$ & 2102 & 204853.05 & +001128.0 & APO & 2005 Sep 27 \\
\hline $2005 \mathrm{fr}$ & 2165 & 010822.02 & -000546.7 & Subaru & 2005 Sep 29 \\
\hline 2005 fy & 2246 & 032021.70 & -005308.1 & APO & 2005 Sep 25 \\
\hline 2005 fy & 2246 & 032021.70 & -005308.1 & WHT & 2005 Sep 25 \\
\hline 2005 ey & 2308 & 021705.50 & +001648.9 & WHT & 2005 Sep 23 \\
\hline $2005 \mathrm{fp}$ & 2330 & 002713.69 & +010714.2 & Subaru & 2005 Sep 28 \\
\hline \multirow[t]{2}{*}{$2005 \mathrm{fq}$} & 2366 & 005344.05 & -003336.8 & HET & 2005 Oct 01 \\
\hline & 2371 & 023958.49 & -00 3149.4 & APO & 2005 Nov 06 \\
\hline $2005 \mathrm{ft}$ & 2372 & 024204.98 & -003227.0 & WHT & 2005 Sep 24 \\
\hline $2005 \mathrm{ft}$ & 2372 & 024204.98 & -003227.0 & APO & 2005 Sep 25 \\
\hline $2005 \mathrm{fi}$ & 2422 & 000758.69 & +0038 17.4 & Subaru & 2005 Sep 28 \\
\hline $2005 f u$ & 2440 & 025032.09 & +004828.1 & WHT & 2005 Sep 24 \\
\hline $2005 \mathrm{fs}$ & 2533 & 020452.98 & -001935.2 & HET & 2005 Oct 01 \\
\hline $2005 \mathrm{fv}$ & 2561 & 030522.42 & +005130.1 & MDM & 2005 Sep 24 \\
\hline $2005 \mathrm{fv}$ & 2561 & 030522.42 & +005130.1 & WHT & 2005 Sep 24 \\
\hline $2005 \mathrm{fv}$ & 2561 & 030522.42 & +005130.1 & APO & 2005 Sep 25 \\
\hline $2005 \mathrm{fv}$ & 2561 & 030522.42 & +005130.1 & MDM & 2005 Sep 25 \\
\hline $2005 \mathrm{fw}$ & 2635 & 033049.04 & -011417.2 & WHT & 2005 Sep 25 \\
\hline \multirow[t]{2}{*}{$2005 \mathrm{fw}$} & 2635 & 033049.04 & -011417.2 & Subaru & 2005 Oct 27 \\
\hline & 2661 & 233249.81 & +000549.9 & Subaru & 2005 Sep 28 \\
\hline $2005 \mathrm{fa}$ & 2689 & 013936.09 & -004531.5 & WHT & 2005 Sep 23 \\
\hline \multirow[t]{3}{*}{$2005 \mathrm{hm}$} & 2744 & 213900.65 & -010138.7 & MDM & 2005 Oct 24 \\
\hline & 2746 & 021825.13 & +010800.9 & APO & 2005 Sep 25 \\
\hline & 2746 & 021825.13 & +010800.9 & WHT & 2005 Sep 26 \\
\hline \multirow[t]{2}{*}{$2005 f x$} & 2789 & 225648.34 & +002403.9 & Subaru & 2005 Sep 27 \\
\hline & 2864 & 235748.24 & -011422.6 & HET & 2005 Dec 22 \\
\hline $2005 \mathrm{fz}$ & 2916 & 210341.22 & +003410.3 & WHT & 2005 Sep 26 \\
\hline $2005 \mathrm{fz}$ & 2916 & 210341.22 & +00 3410.3 & APO & 2005 Sep 27 \\
\hline $2005 \mathrm{fz}$ & 2916 & 210341.22 & +003410.3 & APO & 2005 Oct 01 \\
\hline
\end{tabular}


Table 1

(Continued)

\begin{tabular}{|c|c|c|c|c|c|}
\hline $\begin{array}{l}\text { IAU }^{\mathrm{a}} \\
\text { Name }\end{array}$ & SNID $^{b}$ & $\begin{array}{c}\text { R.A. } \\
\text { (J2000) }\end{array}$ & $\begin{array}{c}\text { Decl. } \\
(\mathrm{J} 2000)\end{array}$ & Telescope & $\begin{array}{c}\text { Obs. Date } \\
\text { (UT) }\end{array}$ \\
\hline & 2928 & 001924.60 & -003520.8 & HET & 2005 Dec 29 \\
\hline$\ldots$ & 2929 & 003602.88 & -005006.0 & MDM & 2005 Sep 25 \\
\hline 2005go & 2943 & 011049.18 & +010028.4 & HET & 2005 Oct 09 \\
\hline $2005 g p$ & 2992 & 034159.29 & -004657.6 & APO & 2005 Oct 07 \\
\hline $2005 g p$ & 2992 & 034159.29 & -004657.6 & Subaru & 2005 Oct 26 \\
\hline $2005 \mathrm{ga}$ & 3080 & 010743.76 & -010222.2 & WHT & 2005 Sep 26 \\
\hline $2005 \mathrm{ga}$ & 3080 & 010743.76 & -010222.2 & APO & 2005 Sep 28 \\
\hline 2005ga & 3080 & 010743.76 & -010222.2 & Subaru & 2005 Oct 27 \\
\hline $2005 \mathrm{gc}$ & 3087 & 012137.62 & -005838.0 & MDM & 2005 Oct 03 \\
\hline $2005 \mathrm{gs}$ & 3199 & 221310.25 & +010302.1 & HET & 2005 Oct 09 \\
\hline $2005 \mathrm{gh}$ & 3241 & 205036.35 & -002114.8 & WHT & 2005 Sep 30 \\
\hline $2005 \mathrm{hn}$ & 3256 & 215704.23 & -001324.4 & MDM & 2005 Oct 25 \\
\hline $2005 \mathrm{gd}$ & 3317 & 014751.03 & +003826.2 & APO & 2005 Sep 27 \\
\hline 2005ge & 3331 & 021814.73 & +004747.6 & WHT & 2005 Sep 26 \\
\hline $2005 \mathrm{gr}$ & 3377 & 033637.49 & +010445.0 & HET & 2005 Oct 12 \\
\hline $2005 \mathrm{gf}$ & 3451 & 221616.61 & +004229.5 & Subaru & 2005 Sep 27 \\
\hline $2005 \mathrm{gg}$ & 3452 & 221841.16 & +003821.1 & Subaru & 2005 Sep 27 \\
\hline$\ldots$ & 3508 & 010317.71 & -001044.4 & HET & 2005 Dec 20 \\
\hline$\cdots$ & 3508 & 010317.71 & -001044.4 & HET & 2005 Dec 25 \\
\hline$\ldots$ & 3535 & 025628.13 & -000800.5 & HET & 2005 Dec 21 \\
\hline $2005 \mathrm{gb}$ & 3592 & 011612.58 & +004731.0 & APO & 2005 Sep 27 \\
\hline $2005 \mathrm{gb}$ & 3592 & 011612.58 & +004731.0 & MDM & 2005 Oct 01 \\
\hline $2005 \mathrm{gb}$ & 3592 & 011612.58 & +004731.0 & MDM & 2005 Oct 13 \\
\hline $2005 \mathrm{gi}$ & 3818 & 005552.68 & +003017.8 & $\mathrm{APO}$ & 2005 Sep 27 \\
\hline 2005ho & 3901 & 005924.10 & +000009.3 & APO & 2005 Sep 28 \\
\hline 2005ho & 3901 & 005924.10 & +000009.3 & MDM & 2005 Oct 20 \\
\hline$\ldots$ & 3959 & 030023.50 & +003042.8 & HET & 2005 Dec 30 \\
\hline$\ldots$ & 4000 & 020403.84 & -002156.9 & HET & 2005 Oct 10 \\
\hline $2005 \mathrm{gt}$ & 4012 & 031347.74 & -001437.0 & WHT & 2005 Oct 24 \\
\hline $2005 \mathrm{gw}$ & 4046 & & +003831.7 & HET & 2005 Oct 10 \\
\hline$\cdots$ & 4064 & 030426.32 & +010247.1 & APO & 2005 Oct 07 \\
\hline$\ldots$ & 4236 & 000737.36 & -010106.0 & HET & 2005 Dec 23 \\
\hline $2005 \mathrm{gu}$ & 4241 & 004857.05 & -005420.8 & HET & 2005 Oct 10 \\
\hline$\ldots$ & 4281 & 021328.18 & -005805.7 & APO & 2005 Oct 05 \\
\hline$\ldots$ & 4307 & 015951.00 & -005658.9 & HET & 2005 Dec 21 \\
\hline$\ldots$ & 4311 & 020831.43 & +010111.9 & HET & 2005 Dec 22 \\
\hline $2005 \mathrm{gj}$ & 4524 & 030111.96 & -003313.9 & MDM & 2005 Oct 01 \\
\hline $2005 \mathrm{gj}$ & 4524 & 030111.96 & -003313.9 & APO & 2005 Oct 03 \\
\hline $2005 \mathrm{gj}$ & 4524 & 030111.96 & -003313.9 & APO & 2005 Oct 07 \\
\hline $2005 \mathrm{gj}$ & 4524 & 030111.96 & -003313.9 & MDM & 2005 Oct 12 \\
\hline $2005 \mathrm{gj}$ & 4524 & 030111.96 & -003313.9 & MDM & 2005 Oct 22 \\
\hline $2005 \mathrm{gj}$ & 4524 & 030111.96 & -003313.9 & MDM & 2005 Oct 25 \\
\hline $2005 \mathrm{gj}$ & 4524 & 030111.96 & -003313.9 & MDM & 2005 Nov 02 \\
\hline $2005 \mathrm{gj}$ & 4524 & 030111.96 & -003313.9 & WHT & 2005 Nov 10 \\
\hline $2005 \mathrm{gj}$ & 4524 & 030111.96 & -003313.9 & MDM & 2005 Nov 12 \\
\hline $2005 \mathrm{gj}$ & 4524 & 030111.96 & -003313.9 & WHT & 2005 Nov 12 \\
\hline $2005 \mathrm{gj}$ & 4524 & 030111.96 & -003313.9 & MDM & 2005 Nov 26 \\
\hline $2005 \mathrm{gj}$ & 4524 & 030111.96 & -003313.9 & MDM & 2005 Nov 28 \\
\hline $2005 \mathrm{gj}$ & 4524 & 030111.96 & -003313.9 & MDM & 2005 Dec 08 \\
\hline $2005 \mathrm{gj}$ & 4524 & 030111.96 & -003313.9 & MDM & 2005 Dec 25 \\
\hline $2005 \mathrm{gv}$ & 4577 & 023354.13 & +001650.6 & HET & 2005 Oct 08 \\
\hline$\ldots$ & 4676 & 011517.68 & +004717.5 & HET & 2005 Dec 21 \\
\hline $2005 g y$ & 4679 & 012606.79 & +004036.8 & HET & 2005 Oct 08 \\
\hline $2005 g x$ & 5103 & 235932.27 & +004413.7 & APO & 2005 Oct 05 \\
\hline $2005 \mathrm{gq}$ & 5183 & 033348.97 & +004233.7 & HET & 2005 Oct 08 \\
\hline $2005 \mathrm{hp}$ & 5350 & 202852.60 & -004645.4 & HET & 2005 Oct 22 \\
\hline$\ldots$ & 5378 & 023056.86 & -011507.5 & MDM & 2005 Dec 26 \\
\hline $2005 \mathrm{hs}$ & 5391 & 032922.06 & -010541.0 & Subaru & 2005 Oct 26 \\
\hline $2005 \mathrm{hr}$ & 5395 & 031833.81 & +000724.3 & MDM & 2005 Oct 23 \\
\hline 2005hu & 5533 & 215440.80 & +002447.8 & Subaru & 2005 Oct 26 \\
\hline $2005 h x$ & 5549 & 001300.13 & +001453.7 & MDM & 2005 Oct 22 \\
\hline 2005hy & 5550 & 001423.59 & +00 1959.1 & WHT & 2005 Oct 24 \\
\hline $2005 \mathrm{hw}$ & 5588 & 000928.47 & +010917.6 & HET & 2005 Oct 27 \\
\hline $2005 \mathrm{hv}$ & 5635 & 221243.86 & -000205.6 & WHT & 2005 Oct 24 \\
\hline
\end{tabular}

Table 1

(Continued)

\begin{tabular}{|c|c|c|c|c|c|}
\hline $\begin{array}{l}\text { IAU }^{\mathrm{a}} \\
\text { Name }\end{array}$ & $\mathrm{SNID}^{\mathrm{b}}$ & $\begin{array}{c}\text { R.A. } \\
\text { (J2000) }\end{array}$ & $\begin{array}{c}\text { Decl. } \\
(\mathrm{J} 2000)\end{array}$ & Telescope & $\begin{array}{c}\text { Obs. Date } \\
\text { (UT) }\end{array}$ \\
\hline & 5673 & 233512.32 & & HET & 2005 Oct 26 \\
\hline $2005 i a$ & 5717 & 011135.02 & -000021.0 & Subaru & 2005 Oct 27 \\
\hline $2005 j \mathrm{z}$ & 5736 & 013127.07 & -003754.0 & HET & 2005 Nov 05 \\
\hline $2005 \mathrm{ib}$ & 5737 & 013125.70 & -003612.3 & Subaru & 2005 Oct 27 \\
\hline $2005 \mathrm{hz}$ & 5751 & 004632.18 & +005018.1 & WHT & 2005 Oct 24 \\
\hline $2005 \mathrm{hz}$ & 5751 & 004632.18 & +005018.1 & Subaru & 2005 Nov 27 \\
\hline $2005 \mathrm{hq}$ & 5821 & 205019.78 & -004931.0 & HET & 2005 Oct 25 \\
\hline 2005hq & 5821 & 205019.78 & -004931.0 & HET & 2005 Oct 27 \\
\hline $2005 \mathrm{ic}$ & 5844 & 215108.69 & -005034.6 & Subaru & 2005 Oct 26 \\
\hline 2005 is & 5916 & 002144.97 & -001930.0 & MDM & 2005 Oct 25 \\
\hline 2005is & 5916 & 002144.97 & -001930.0 & APO & 2005 Oct 31 \\
\hline $2005 \mathrm{hc}$ & 5944 & 015647.94 & -001249.1 & MDM & 2005 Oct 22 \\
\hline $2005 \mathrm{hc}$ & 5944 & 015647.94 & -001249.1 & MDM & 2005 Oct 24 \\
\hline $2005 \mathrm{hc}$ & 5944 & 015647.94 & & Subaru & 2005 Nov 27 \\
\hline 2005 ie & 5957 & 021902.53 & -001622.0 & Subaru & 2005 Oct 26 \\
\hline & 5963 & 00441 & +002 & HET & 2005 Dec 23 \\
\hline 2005 it & 5966 & 01044 & & & $2005 \mathrm{Oc}$ \\
\hline$\ldots$ & 5993 & 015843. & & & \\
\hline $2005 \mathrm{ht}$ & 5994 & 205024.60 & -00 & WHT & 2005 Oct 24 \\
\hline 2005 if & 6057 & 033012.87 & & & 2005 Oct 23 \\
\hline 2005 if & 6057 & 033012. & -005 & & 2005 Nov 26 \\
\hline $2005 \mathrm{ka}$ & 6100 & 22135 & +0 & HET & 2005 Nov 04 \\
\hline 2005ih & 6108 & 00071 & & aru & 2005 Oct 28 \\
\hline 2005 iw & 6127 & 22291 & & ET & 5 Oct 30 \\
\hline 2005 iv & 6137 & 20314 & & ET & $2005 \mathrm{~N}$ \\
\hline 2005jy & 6192 & 23135 & & ET & 05 \\
\hline $2005 i g$ & 6196 & 223031. & -00 & Subaru & 2005 Oct 27 \\
\hline $2005 \mathrm{ii}$ & 6249 & & & & $2005 \mathrm{Oc}$ \\
\hline & & & & & \\
\hline $2005 \mathrm{jk}$ & 6304 & 01455 & +01 & $\mathrm{PO}$ & $2005 \mathrm{Nc}$ \\
\hline $2005 \mathrm{jk}$ & 6304 & 3 & & & $2005 \mathrm{Nc}$ \\
\hline & & & & & \\
\hline $2005 i x$ & 6315 & 204155.88 & +01 & $\mathrm{ck}$ & v 05 \\
\hline $2005 \mathrm{ij}$ & 6406 & 030421.26 & & VHT & 2005 Oct 24 \\
\hline $2005 \mathrm{ij}$ & 6406 & & -01 & & 2005 Nov 26 \\
\hline 2005id & 6422 & 23163 & -00 & WHT & et 24 \\
\hline$\ldots$ & 6471 & 203732. & +00 & Subaru & 2005 Oct 28 \\
\hline $2005 \mathrm{hj}^{\mathrm{c}}$ & & 012648.40 & -01 & & 2005 Oct 26 \\
\hline $2005 \mathrm{jd}$ & 6649 & 02170 & & ET & 200 \\
\hline $2005 \mathrm{jd}$ & & 021706.21 & +00 & Keck & 2005 Nov 05 \\
\hline & & 223619.04 & & & 2005 Oct 31 \\
\hline 2005 ik & 6699 & 213115.60 & -010325.2 & Subaru & 2005 Oct 28 \\
\hline 2005ik & & & & & \\
\hline$\ldots$ & 6714 & 234926.4 & & & 2005 Dec 20 \\
\hline 2005iu & 6773 & 202015.60 & +00 & APO & 2005 Oct 31 \\
\hline 2005iy & 6777 & & +00 & & $2005 \mathrm{Oc}$ \\
\hline 2005iy & 6777 & 212451.94 & & & 2005 Nov 02 \\
\hline $2005 \mathrm{iz}$ & 6780 & 215216.47 & +00 & HET & 2005 Nov 01 \\
\hline 2005jf & & 033340.76 & & & 2005 Oct 31 \\
\hline $2005 \mathrm{ja}$ & 6924 & 235552.64 & +00 & & 2005 Oct 31 \\
\hline $2005 j \mathrm{c}$ & 6933 & 004524.41 & +010432.1 & APO & 2005 Nov 02 \\
\hline $2005 \mathrm{jc}$ & & 004524.41 & & & 2005 Nov 05 \\
\hline 2005jl & 6936 & 213256.12 & -004159.2 & & 2005 Nov 05 \\
\hline 2005je & 6962 & 023526.61 & +010429.6 & APO & 2005 Nov 02 \\
\hline & 6968 & 011813.37 & & APO & 2005 Nov 24 \\
\hline$\ldots$ & 7017 & 020527.68 & -002952.5 & APO & 2005 Nov 02 \\
\hline$\ldots$ & 7099 & 225133.17 & +011002.5 & APO & 2005 Oct 31 \\
\hline$\ldots$ & 7102 & 213828.78 & & APO & 2005 Oct 31 \\
\hline 2005jg & 7143 & 230102.97 & -001226.7 & HET & 2005 Nov 01 \\
\hline 2005jh & 7147 & 232004.42 & -000319.8 & MDM & 2005 Nov 03 \\
\hline $2005 \mathrm{jh}$ & 7147 & 232004.42 & -000319.8 & & 2005 Nov 05 \\
\hline $2005 \mathrm{jm}$ & 7243 & 215218.97 & +002819.1 & Keck & 2005 Nov 05 \\
\hline $2005 \mathrm{kn}$ & 7335 & 211532.45 & -002119.2 & HET & 2005 Nov 09 \\
\hline $2005 \mathrm{jb}$ & 7426 & 223603.21 & -002204.5 & HET & 2005 Oct 31 \\
\hline
\end{tabular}


Table 1

(Continued)

\begin{tabular}{|c|c|c|c|c|c|}
\hline $\begin{array}{l}\text { IAU }^{\mathrm{a}} \\
\text { Name }\end{array}$ & SNID $^{b}$ & $\begin{array}{c}\text { R.A. } \\
(\mathrm{J} 2000)\end{array}$ & $\begin{array}{c}\text { Decl. } \\
(\mathrm{J} 2000)\end{array}$ & Telescope & $\begin{array}{c}\text { Obs. Date } \\
\text { (UT) }\end{array}$ \\
\hline & 7444 & 015048.71 & -002547.4 & HET & 2005 Dec 19 \\
\hline $2005 j x$ & 7460 & 213420.18 & -004054.0 & HET & 2005 Nov 04 \\
\hline $2005 \mathrm{ji}$ & 7473 & 001718.34 & -001526.1 & MDM & 2005 Nov 04 \\
\hline $2005 \mathrm{ji}$ & 7473 & 001718.34 & -001526.1 & Keck & 2005 Nov 05 \\
\hline 2005jn & 7475 & 001900.84 & -001653.3 & HET & 2005 Nov 05 \\
\hline 2005jn & 7475 & 001900.84 & -001653.3 & Keck & 2005 Nov 05 \\
\hline 2005jo & 7512 & 032821.68 & -001934.1 & Keck & 2005 Nov 05 \\
\hline$\cdots$ & 7647 & 235331.95 & +010231.3 & HET & 2005 Dec 21 \\
\hline $2005 \mathrm{jw}$ & 7779 & 204019.25 & -000025.9 & HET & 2005 Nov 05 \\
\hline 2005jp & 7847 & 020950.38 & -000342.2 & Keck & 2005 Nov 05 \\
\hline $2005 \mathrm{ir}$ & 7876 & 011643.80 & +004740.7 & APO & 2005 Nov 02 \\
\hline $2005 \mathrm{ir}$ & 7876 & 011643.80 & +004740.7 & APO & 2005 Nov 06 \\
\hline$\ldots$ & 7922 & 021253.30 & -005503.1 & HET & 2005 Nov 02 \\
\hline 2005jj & 7947 & 205644.66 & +002430.0 & HET & 2005 Nov 03 \\
\hline $2005 \mathrm{jv}$ & 8030 & 024050.12 & +005935.2 & HET & 2005 Nov 05 \\
\hline 2005ju & 8046 & 023628.04 & +003040.6 & HET & 2005 Nov 04 \\
\hline $2005 \mathrm{hk}$ & 8151 & 002750.89 & -011153.3 & MDM & 2005 Nov 03 \\
\hline $2005 \mathrm{hk}$ & 8151 & 002750.89 & -011153.3 & APO & 2005 Nov 04 \\
\hline 2005hk & 8151 & 002750.89 & -011153.3 & MDM & 2005 Nov 04 \\
\hline $2005 \mathrm{hk}$ & 8151 & 002750.89 & -011153.3 & APO & 2005 Nov 06 \\
\hline $2005 \mathrm{hk}$ & 8151 & 002750.89 & -011153.3 & WHT & 2005 Nov 10 \\
\hline $2005 \mathrm{hk}$ & 8151 & 002750.89 & -011153.3 & WHT & 2005 Nov 12 \\
\hline $2005 \mathrm{hk}$ & 8151 & 002750.89 & -011153.3 & MDM & 2005 Nov 14 \\
\hline $2005 \mathrm{hk}$ & 8151 & 002750.89 & -011153.3 & MDM & 2005 Dec 07 \\
\hline 2005ko & 8213 & 235005.03 & -005517.1 & HET & 2005 Nov 21 \\
\hline $2005 \mathrm{mi}$ & 8495 & 222102.65 & -004453.4 & HET & 2005 Dec 07 \\
\hline $2005 \mathrm{jt}$ & 8598 & 025040.17 & -000357.5 & HET & 2005 Nov 06 \\
\hline $2005 \mathrm{jr}$ & 8679 & 010012.37 & +002026.9 & Keck & 2005 Nov 05 \\
\hline $2005 \mathrm{mh}$ & 8707 & 024456.67 & +001213.2 & HET & 2005 Dec 07 \\
\hline $2005 \mathrm{kp}$ & 8719 & & -004307.9 & APO & \\
\hline$\ldots$ & 8742 & 004457.49 & +002614.2 & HET & 2005 Dec 31 \\
\hline 2005ld & 8921 & 214000.47 & -000028.5 & APO & 2005 Nov 24 \\
\hline 2005le & 9032 & 223132.29 & -002936.8 & Subaru & 2005 Nov 26 \\
\hline $2005 \mathrm{kq}$ & 9045 & 231120.91 & -003631.0 & HET & 2005 Nov 22 \\
\hline $2005 \mathrm{kr}$ & 9118 & 030829.67 & +005320.2 & HET & 2005 Nov 22 \\
\hline $2005 \lg$ & 9207 & 011620.07 & -004828.1 & Subaru & 2005 Nov 26 \\
\hline $2005 \mathrm{ks}$ & 9273 & 213756.56 & -000156.9 & HET & 2005 Nov 22 \\
\hline 2005li & 9457 & 222315.45 & +001511.0 & HET & 2005 Nov 23 \\
\hline 2005lh & 9467 & 215548.33 & +011051.0 & HET & 2005 Dec 01 \\
\hline$\ldots$ & 9954 & 002620.19 & -002531.6 & HET & 2005 Dec 26 \\
\hline $2005 \mathrm{kt}$ & 10028 & 011058.04 & +001634.2 & WHT & 2005 Nov 12 \\
\hline$\ldots$ & 10045 & 214846.84 & +011241.2 & HET & 2005 Nov 27 \\
\hline $20051 \mathrm{j}$ & 10096 & 015743.04 & -001046.0 & APO & 2005 Nov 26 \\
\hline$\ldots$ & 10106 & 031046.39 & -001217.6 & HET & 2005 Dec 28 \\
\hline $2005 \mathrm{mk}$ & 10297 & 224240.63 & +004958.9 & HET & 2005 Dec 04 \\
\hline $2005 \mathrm{~kb}$ & 10367 & 005050.68 & +005113.0 & APO & 2005 Nov 09 \\
\hline 2005lk & 10434 & 215949.42 & -011137.4 & APO & 2005 Nov 24 \\
\hline 200511 & 10449 & 222806.88 & -010741.6 & Subaru & 2005 Nov 27 \\
\hline 2005lf & 10550 & 231842.09 & -011217.6 & Subaru & 2005 Nov 26 \\
\hline $2005 \mathrm{ku}$ & 10805 & 225942.61 & -000049.4 & MDM & 2005 Nov 22 \\
\hline $20051 \mathrm{~m}$ & 10907 & 001504.91 & +002118.6 & APO & 2005 Nov 24 \\
\hline$\ldots$ & 10915 & 012152.25 & +002319.9 & HET & 2005 Dec 23 \\
\hline$\cdots$ & 10928 & 002642.53 & -004813.8 & APO & 2005 Nov 26 \\
\hline$\ldots$ & 10976 & 012840.94 & -005321.2 & APO & 2005 Nov 26 \\
\hline $2005 \mathrm{mj}^{\mathrm{d}}$ & 11017 & 213149.44 & -010409.9 & Magellan & 2005 Nov 19 \\
\hline $2005 \mathrm{ml}$ & 11067 & 021404.41 & -001421.0 & APO & 2005 Dec 04 \\
\hline $2005 \mathrm{~mm}$ & 11206 & 001309.55 & +010843.9 & HET & 2005 Dec 03 \\
\hline $2005 \ln$ & 11300 & 002700.12 & -003511.8 & APO & 2005 Nov 24 \\
\hline $2005 \mathrm{mo}$ & 11320 & 035012.90 & -001424.9 & HET & 2005 Dec 07 \\
\hline $2005 \mathrm{mn}$ & 11332 & 034918.44 & -004131.5 & HET & 2005 Dec 01 \\
\hline $2005 \mathrm{mn}$ & 11332 & 034918.44 & -004131.5 & APO & 2005 Dec 04 \\
\hline $2005 \mathrm{mn}$ & 11332 & 034918.44 & -004131.5 & HET & 2005 Dec 07 \\
\hline $2005 \mathrm{mn}$ & 11332 & 034918.44 & -004131.5 & HET & 2005 Dec 30 \\
\hline 2005lo & 11452 & 003711.87 & -011212.4 & HET & 2005 Dec 02 \\
\hline
\end{tabular}

Table 1

(Continued)

\begin{tabular}{lccccc}
\hline \hline $\begin{array}{l}\text { IAU }^{\mathrm{a}} \\
\text { Name }\end{array}$ & SNID $^{\mathrm{b}}$ & $\begin{array}{c}\text { R.A. } \\
(\mathrm{J} 2000)\end{array}$ & $\begin{array}{c}\text { Decl. } \\
(\mathrm{J} 2000)\end{array}$ & Telescope & $\begin{array}{c}\text { Obs. Date } \\
(\mathrm{UT})\end{array}$ \\
\hline $2005 \mathrm{lq}$ & 11557 & 024136.05 & +001218.0 & HET & 2005 Dec 01 \\
2005mp & 11650 & 010445.67 & +000320.3 & HET & 2005 Dec 03 \\
$2005 \mathrm{lp}$ & 11864 & 014742.80 & +001225.9 & HET & 2005 Dec 01 \\
$2005 \mathrm{mq}$ & 12136 & 232021.78 & -002059.6 & HET & 2005 Dec 07 \\
\hline
\end{tabular}

Notes.

a Official IAU name, when assigned.

b SDSS Internal SN candidate ID.

c Spectroscopically confirmed by another group using the HET (CBET 266; Quimby et al. 2007). The spectrum is not available.

${ }^{\mathrm{d}}$ Spectroscopically confirmed by the ESSENCE group.

the slit width was set at $1^{\prime \prime}$, providing a resolution of $4.3 \AA$ in the blue and $7.5 \AA$ in the red, although the slit was adjusted to accommodate variable seeing. For the observations in October and November, a GG495 filter was employed in the red channel to eliminate second-order contamination. The observations provided $26 \mathrm{SNe}$ Ia, three probable $\mathrm{SNe}$ Ia, and one $\mathrm{SN}$ Ic (with a median $\mathrm{SN}$ redshift of $\sim 0.16$ ).

\subsubsection{Subaru $8.2 \mathrm{~m}$}

At this telescope we used the Faint Object Camera and Spectrograph (FOCAS), taking advantage of the excellent image quality to employ a $0.8^{\prime \prime}$ slit and target higher-redshift $\mathrm{SNe}$ with significant host-galaxy contamination. Separate exposures were made using blue and red $3001 \mathrm{~mm}^{-1}$ grisms. The blue setting covered 3650-6000 A, while the red covered 4900-9000 $\AA$. The resulting resolution was $\sim 8-12 \AA$. Typical exposures of $3 \times 300 \mathrm{~s}$ allowed us to confirm $30 \mathrm{SNe}$ Ia, one probable SN Ia, and two $\mathrm{SNe}$ II, with a median redshift of $\sim 0.25$.

\subsubsection{Keck $10 \mathrm{~m}$}

One target-of-opportunity night (2005 November 5) was obtained with the Low-Resolution Imaging Spectrograph (LRIS; Oke et al. 1995) on Keck I. The blue side covered 3200-5700 ̊ with a $6001 \mathrm{~mm}^{-1}$ grating (4000 $\AA$ blaze; $1.92 \AA$ pixel $^{-1}$ dispersion), and the red side covered $\sim 5500-9400 \AA$ with a $4001 \mathrm{~mm}^{-1}$ grating (8500 ̊ blaze; $0.61 \AA \mathrm{p} \mathrm{pixel}^{-1}$ dispersion). A slit of width 1" was oriented at the parallactic angle for an effective resolution of $8.9 \AA$ (blue) and $4.5 \AA$ (red). With exposures ranging from 700 to $2400 \mathrm{~s}$, we observed $12 \mathrm{SNe}$ Ia and one SN IIn; the median redshift was $\sim 0.24$. Most of these objects had been previously confirmed as spectroscopic SNe Ia at our other telescopes, but the Keck spectra generally had higher signal-to-noise ratio $(\mathrm{S} / \mathrm{N})$ and provided important rest-frame UV information on the SNe.

\subsection{Calibrations and Reductions}

Basic data calibration (bias/overscan subtraction, flux calibration, and wavelength calibration) was performed by the individual observing teams using standard IRAF ${ }^{49}$ routines. SN spectra were extracted from the geometrically corrected twodimensional (2D) spectra, often with significant amounts of

49 IRAF is distributed by the National Optical Astronomy Observatory, which is operated by the Association of Universities for Research in Astronomy, Inc., under cooperative agreement with the National Science Foundation. 


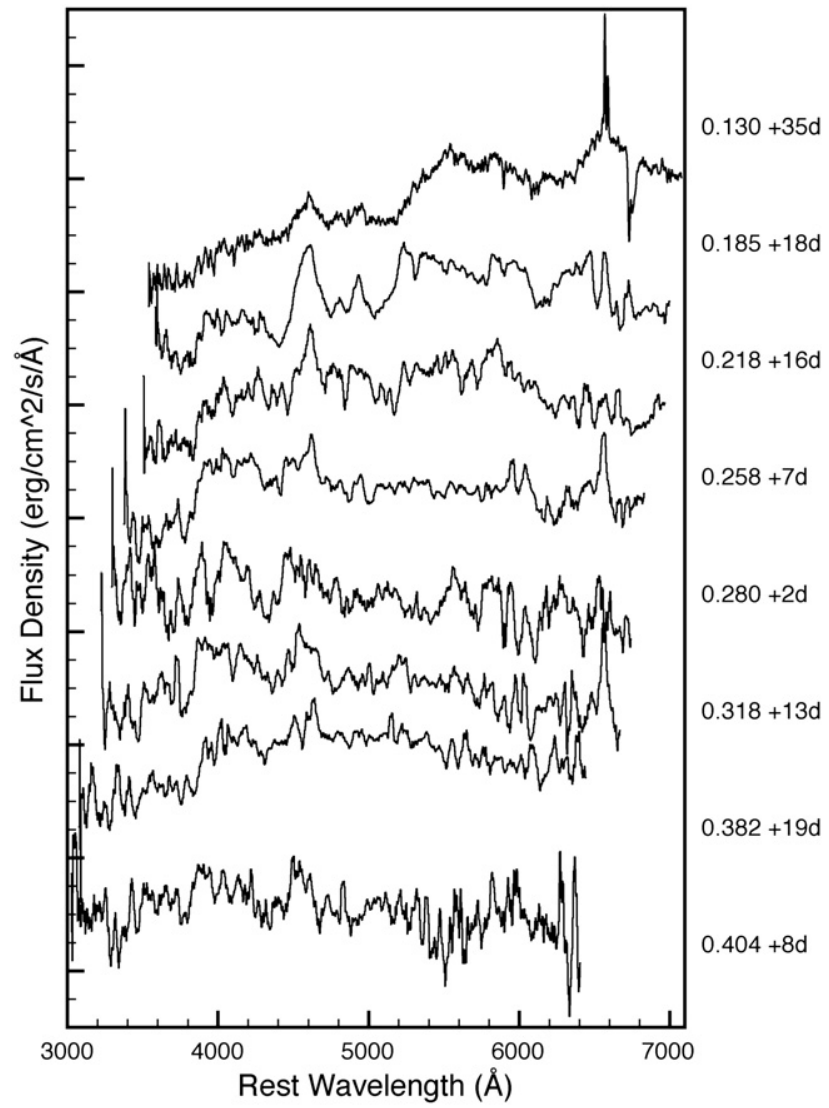

Figure 3. A sample of observed Ia spectra from the fall 2005 run with significan host-galaxy contamination. All spectra have been shifted to the rest frame (numbers at right give the redshifts and epochs relative to $B$-band maximum) and have been normalized for display purposes. The top spectrum (from Subaru) has not been corrected for telluric absorption.

host-galaxy background (see Figure 3); for higher- $z$ targets embedded in their hosts, modest spatial-width apertures were used in extracting the SN spectra to minimize host contamination. In half of the cases, a separate host-dominated aperture could be extracted from the 2D spectrum to produce a host-galaxy spectrum. For ARC observations, a separate host-core aperture was often extracted. For the other observations, the near parallactic orientation minimized chromatic slit losses; however, given our narrow slits and extraction apertures, no attempt at absolute spectrophotometry was made. Reductions of the HET, ARC, WHT, and Keck data included a correction for telluric absorption.

\section{SN IDENTIFICATION}

The principal goal of the spectroscopic observations is to classify the SN and measure its redshift. We can also use these measurements to improve the choice of future spectroscopic targets. For low- $z$ SNe with high-S/N spectra, the classification is, in principle, simple (Filippenko 1997)-Type II show strong Balmer lines, Type Ia show strong Si II lines, Type Ib lack $\mathrm{H}$ lines but show strong He emission, Type Ic lack all of the above. Traditional classification is done by visual inspection. In practice, however, our spectra have a widely varying $\mathrm{S} / \mathrm{N}$, cover a large redshift range, and suffer significant host-galaxy contamination and extinction. Further, with this large, relatively unbiased sample, we expect rare, "peculiar" SNe to be present (which are not discussed further in the present paper, but will be part of a future analysis with the complete spectroscopic sample). We have sought to develop automatic classification programs capable of dealing with our sample, exploring template cross-correlation and principal component analysis (PCA) techniques. While these have substantial discriminating power and also serve to find best-fitted parameters and their associated statistical errors, the final object (SN- and host-type) classification required some human judgment in at least half of the cases.

Of course, we also need to obtain the accurate redshifts required when using the $\mathrm{SNe}$ for cosmology and population studies. These were robustly measured in the cross-correlation analysis, especially in the $\sim 50 \%$ of the spectra with large host-galaxy contamination or separate host spectra. These are augmented by "host-only" spectra, obtained for SNe that had faded before spectroscopy could be arranged (these sources are not discussed further in the present paper). When cross correlation could be locked to the narrow-line host features, we refer to the result as a "galaxy redshift;" these have a typical error of $\delta z \approx 0.0005$. In other cases, we could only match the broad blueshifted $\mathrm{SN}$ features; the resulting redshifts $\left(z_{\mathrm{SN}}\right)$ vary in quality, with $\delta z \sim 0.005$ (see discussion below).

In the following sections, we discuss these classification efforts along with our work to model and remove the host-galaxy contamination and extinction. The result is a set of "clean" SN spectra with estimates of the quality of the host subtraction and measurements of the agreement with standard templates of the best-fitting SN type. The latter may be used to flag $\mathrm{SNe}$ with significant spectral anomalies, useful in improving our understanding of SN physics and improving our calibration of the SN luminosities.

\subsection{Cross-Correlation Analysis}

With a suitable set of templates, cross-correlation analysis provides a well-established way to measure redshift and to constrain the spectral type. In the method developed by Tonry \& Davis (1979), one computes the cross correlation $c(n)=s(n) * t(n)$ of the original $s$ and template $t$ spectra at zero redshift, after scaling the wavelength axis of $t(n)$ by a factor of $(1+z)$. First the spectra are continuum subtracted, $\ln (\lambda)$ binned, endmasked, and filtered to remove any intrinsic color dependence, low-frequency spectral variations, and highfrequency noise beyond the resolution. The data are trimmed to a range appropriate to each telescope/spectrograph combination to avoid the low-S/N ends and regions where sky-subtraction errors dominate (e.g., we truncate HET spectra at $8300 \AA$ ). The code determines the wavelength shift from a fit to the correlation peak; a measure of the fit quality comes from $r$, the ratio of peak height $h$ to the root-mean square (rms), and $\sigma_{a}$, of the antisymmetric component of $c(n)$ about the correlation redshift. For sufficiently large $r$ (typically $>3$; Tonry \& Davis 1979; Blondin et al. 2007; Blondin \& Tonry 2007), and sufficiently large overlap of $s$ and $t$ in wavelength (defined as the portion of spectrum used for cross correlation; Blondin et al. 2007; Blondin \& Tonry 2007), the measurement of the shift is deemed significant.

In our analysis we use the "rvsao.xcsao" cross-correlation package of IRAF. Our SN templates are from Peter Nugent's spectral library ${ }^{50}$ (see Nugent et al. 2002, and the SUSPECT database ${ }^{51}$ Table 2). Specifically for the $\mathrm{SNe}$ Ia, we used

\footnotetext{
50 http://supernova.lbl.gov/ nugent/nugent_templates.html.

51 http://bruford.nhn.ou.edu/ suspect/index1.html.
} 
Table 2

Cross-Correlation Templates From SUSPECT SN Database

\begin{tabular}{lc}
\hline \hline Type & Object \\
\hline Ibc & 1954A, 1962L, 1964L, 1990B, 1990I, 1994I, 1998bw, 1999ex, 2000H, 2002ap \\
II & 1948B, 1959D, 1961F, 1961I, 1961 V, 1964F, 1964H, 1969L, 1970A, 1986E \\
II & 1987A, 1988A, 1988H, 1988Z, 1989C, 1990ae, 1990ag, 1990E, 1990H, 1990K \\
II & 1990Q, 1990V, 1990X, 1991C, 1991H, 1991J, 1992aa, 1992ab, 1992C, 1992H \\
II & 1993J, 1994aj, 1996L, 1997ab, 1997cy, 1997D, 1998dn, 1998S, 1999em, 2004dj \\
Ia-pec & 1957A, 1960H, 1986G, 1991bg, 1991bj, 1991F, 1991T, 1997br, 1997cn, 1999aa \\
Ia-pec & 1999ac, 1999by, 2000cx \\
\hline
\end{tabular}

Nugent's Branch-normal (Branch et al. 1993), SN 1991T-like (e.g., Filippenko et al. 1992a), and SN 1991bg-like (e.g., Filippenko et al. 1992b) templates (supplemented by spectra of some peculiar SNe Ia (e.g., SN 1999aa, Garavini et al. 2004; SN 1999by, Garnavich et al. 2004, etc) from SUSPECT. For $\mathrm{SNe} \mathrm{Ib} / \mathrm{c}$, the templates used are Nugent's normal SN Ib/c and hypernova spectra, as well as observed spectra of SN 1990B (Matheson et al. 2001), SN 1990I (Elmhamdi et al. 2004), SN 1994I (Clocchiatti et al. 1996), SN 1998bw (Ferdinando et al. 2001), SN 1999ex (Hamuy et al. 2002), SN 2000H (Branch et al. 2002), and SN 2002ap (Gal-Yam et al. 2002), from the SUSPECT database). Similarly, the set of Nugent's SN II-P, SN II-L, and SN IIn spectra, augmented by SUSPECT's SN II and peculiar SN II spectra, constitutes the template library for $\mathrm{SNe}$ II. These templates represent a variety of $\mathrm{SN}$ ages. In particular, the SN Ia templates cover a wide range of epochs ( -19 to $70 \mathrm{~d}$ from peak $B$-band magnitude). Galaxy templates were drawn from the SDSS catalog ${ }^{52}$ and covered five major morphological types: E/S0, Sa, Sb, Sbc/Sc, and Sm/Im.

When fitting for galaxy redshifts $z_{\text {gal }}$, we adjust the xcsao Fourier filtering to focus on narrow features (high frequency in Fourier space). The Fourier cutoff is at a lower frequency when matching SN templates to derive $z_{\mathrm{SN}}$. The cross-correlation program provides a best-fitted redshift, $r$ value, and overlap wavelength range for each template in our library. The relative value of $r$ at the best-fitted redshift provides a guide to the best template class. We discard results with less than $40 \%$ of the spectra in the overlap region (Blondin et al. 2007; Blondin \& Tonry 2007). All measurements are made in the heliocentric frame.

We apply the cross-correlation analysis above to every spectrum obtained and inspect the templates with highest $r$ values and adequate spectral overlap. As expected, the redshift solutions are very insensitive to the template; for example, fits with different galaxy types, such as $\mathrm{Sb}$ and $\mathrm{Sm} / \mathrm{Im}$, give $\delta z \lesssim 0.001$. $\mathrm{SN}$ fits are of course somewhat less precise. Nevertheless, comparing a set of SN Ia and SN Ibc fits gives $\delta z \lesssim 0.005$. Thus, the $z$ measurements reported here are robust. One point deserves comment: half of our spectra have both SN and hostgalaxy redshift solutions, and there is a slight, but statistically significant, systematic offset between the two redshifts (see Figure 4). The difference amounts to $\delta z \sim 0.003$ or a velocity shift of $\sim 900 \mathrm{~km} \mathrm{~s}^{-1}$, which is only a small fraction of the photospheric expansion velocity of the SN $(v \sim$ $10,000 \mathrm{~km} \mathrm{~s}^{-1}$ near maximum light). Different SNe have different expansion velocities (see, e.g., Benetti et al. 2005) and temporal evolution. Since there is no obvious systematic mismatch between the spectroscopic and photometric epochs (Figure 8), we believe the difference is most likely due to the

52 http://www.sdss.org/dr5/algorithms/spectemplates/index.html.

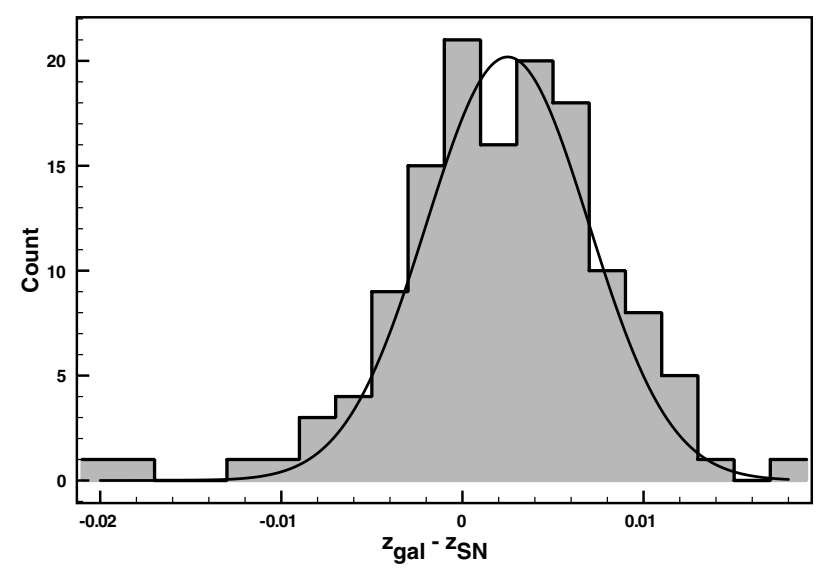

Figure 4. Histogram of $z_{g}-z_{\mathrm{SN}}$ for 136 spectra of $116 \mathrm{SNe}$ in our 2005 data with both host-galaxy and $\mathrm{SN}$ redshift solutions. The red line shows a best-fitted Gaussian, with offset $\delta z=0.003$ and a width of $\sigma_{z}=0.005$. The latter is used as an estimate of the error in the $\mathrm{SN}$ redshift when narrow host-galaxy lines are not detected.

fact that the average photospheric expansion velocity observed in our SN sample is slightly different from that assumed in Nugent's branch-normal SN Ia templates, which is strictly valid for SN Ia with a stretch factor of unity. When only an SN redshift is available, we correct the $z_{\mathrm{SN}}$ for this systematic offset 0.003 (the mean of Figure 4). However, when a significant $r$ is obtained for a galaxy fit $z_{\text {gal }}$, we adopt these more accurate redshifts. The dispersion in the $z_{\mathrm{SN}} / z_{\mathrm{gal}}$ comparison of 0.005 is also adopted here for the systematic error in the $z_{\mathrm{SN}}$ measurement. This error is generally larger than the statistical error $(\delta z \approx 0.001)$ associated with rvsao.xcsao fit results, as it includes systematics associated with uncertainty in the SN velocity and epoch. We compared our galaxy measurements with redshifts from the SDSS database, where available (about a fifth of our sources, primarily at low redshift), finding agreement to within $\delta z=0.001$; no systematic offset is observed.

Comparison of $r$ values between different templates can be used to select the most likely spectral class. For high-quality spectra with little or no host-galaxy contamination, the $r$ values usually cleanly select one SN type. However, for other spectra, we had to visually inspect the solutions at the local $r$ maximum for each SN/galaxy type. This was done by plotting the data over the combined redshifted SN/galaxy spectrum, with a visual normalization, and inspecting how well the features match. Generally, the largest $r$ value was indeed preferred, but in a few cases inspection showed that the largest $r$ corresponded to a misleading fluctuation in the template matches. High-redshift and low-quality spectra, in particular, often showed multiple local minima and human judgment was needed to select the best match. For a small fraction (less than 10\%) of our spectra 
that have very low $\mathrm{S} / \mathrm{N}$ ratio, none of these local minima makes a reasonable fit and thus the spectra were classified as "unknown" and not included in this paper.

We found that $r$ did not select as well between hostgalaxy classes as between $\mathrm{SN}$ types. A clear differentiation between absorption-line-dominated $\mathrm{Sa}$ and emission-linedominated (Irr) spectra is available whenever the $\mathrm{S} / \mathrm{N}$ and host contamination are fairly large. However, finer distinctions (e.g., $\mathrm{Sa} / \mathrm{Sb}$ ) were not reliable. Indeed, for our multi-epoch spectra, $\sim 40 \%$ of the time the best-fitted host template differs by a class between epochs.

We spectroscopically identified SNe Ia according to the following criteria: (1) $r \geqslant 3$ and (2) a high-significance detection of at least one $\mathrm{Si}$ II absorption-line feature (at rest wavelengths of $\sim 4000 \AA, \sim 5800 \AA$, and $\sim 6150 \AA)$. For lower values $(r \approx 3)$, we additionally required a spectrum/template spectral overlap $>60 \%$ (lower overlap values usually indicate a poor type and redshift range) and/or a host with a spectroscopic classification of E/S0. A requirement of $r \geqslant 3$ for SN Ia identification has also been adopted by other groups (Blondin et al. 2007; Blondin $\&$ Tonry 2007; Matheson et al. 2005). When these criteria are satisfied, we designate the SN as Type Ia ("Ia"). When one or more of these criteria fail, but the best match is still a Ia template, we mark the object as "Ia?"- a spectroscopically probable SN Ia. In particular, for late-time spectra the $r$ discrimination was often poor between Types Ia and Ib/c. Occasionally, both SN Ia and SN Ia-pec types gave acceptable fits. In these cases, hostgalaxy type, absolute magnitude, and the extent of the spectral overlap were helpful in making the final classification. Thus, while the redshift estimates are quantitative and, for a given spectrum type, choice of the best-fitted $\mathrm{SN}$ age is quantitative, the selection of the best-type match is necessarily somewhat subjective.

Once a type is confirmed, we find that the $r$ values resolve the SN phase to an accuracy of $\sim 2$ to $3 \mathrm{~d}$, similar to other SN template-fitting techniques (Balland et al. 2006; Blondin et al. 2007; Blondin \& Tonry 2007). The basic results from the cross-correlation analysis are listed in Table 3.

\subsection{Spectral Decomposition and Host-Galaxy Subtraction}

The majority of our SN spectra have substantial host-galaxy contamination (Figures 3 and 5), because of the wide slits used and relatively high redshift and small angular size of the hosts. Indeed, for late-time $\mathrm{SN}$ spectra and subluminous objects, the host-galaxy light coincident with the SN often dominates that of the SN itself. To study SN spectral diversity and to explore the correlation of spectral parameters with our high-quality light curves, we wish to have SN spectra with minimum contamination. Accordingly, we have attempted to quantitatively measure the $\mathrm{SN}$ and host contributions using PCA.

Several approaches have been developed for such spectra decomposition. One method (Madgwick et al. 2003) projects the observed spectrum onto the Karhunen-Loève (KL) transformation or PCA eigenspectra basis (Connolly et al. 1995; Folkes et al. 1996; Madgwick et al. 2002) to extract the reconstructed source (e.g., galaxy) spectrum. Other techniques use point-source deconvolution (Blondin et al. 2005) or $\chi^{2}$ template-fitting techniques (Howell et al. 2002, 2005; Lidman et al. 2005; Hook et al. 2005), modeling both SN and galaxy with template libraries. However, this latter approach sometimes fails, yielding only a low-S/N detection of the $\mathrm{SN}$ spectrum. In such cases, it is common to resort to a crosscorrelation technique similar to that used above (Matheson et al. 2005).

Here we adopt a composite PCA + template-fitting program to separate the $\mathrm{SN}$ spectrum from the host-galaxy light. In this procedure, each observed spectrum is modeled by a weighted combination of SN templates (identical to those used for the cross-correlations analysis) and PCA eigenspectra derived from the SDSS galaxy sample (Yip et al. 2004). The redshift is fixed to the value determined by the cross-correlation procedure above, but we allow the flux from the SN and the three dominant PCA galaxy components to vary. In addition, we optionally add a variable amount of absorption with a Galactic extinction law $A_{\lambda}$ (standard reddening, Cardeli et al. 1989, and $R_{V}=3.1$ ) at the host redshift. To monitor the fit we define a figure of merit (FoM) normalized by the degrees of freedom,

$$
\chi_{\nu}^{2}=\sum_{\lambda} \frac{\left[D(\lambda)-a S(\lambda, T) 10^{c A_{\lambda}}-\sum_{i=0}^{2} b_{i} G_{i}(\lambda)\right]^{2}}{v(\sigma(\lambda))^{2}}
$$

where $D$ is the observed host $+\mathrm{SN}$ spectrum, $S$ is the $\mathrm{SN}$ template spectrum at an epoch $T, G_{0}, G_{1}$, and $G_{2}$ are the first three major PCA eigenspectra of the SDSS galaxy sample (Yip et al. 2004), $A_{\lambda}$ is the extinction (applied at the redshift of the $\mathrm{SN})$, and $\sigma$ is the $1 \sigma$ error of the spectrum. Here $a, b_{0}, b_{1}, b_{2}$, and $c$ are constants to be adjusted for the best fit in the SN spectra, host galaxy, and reddening space. The values of $b_{0}, b_{1}$, and $b_{2}$ indicate the galaxy type of the host (Yip et al. 2004). We neglect extinction and reddening in the Milky Way, which produces $E(B-V)<0.1 \mathrm{mag}$ for almost all of the survey stripe. Here the $v$ degrees of freedom are determined as $N-m$ where $N$ is the number of data points and $m$ accounts for the fitting parameters and the (nearly continuous) date parameter (time from maximum light). Since the first three eigencomponents contain $98.2 \%$ of the total SDSS galaxy sample variance, the amplitudes $b_{0,1,2}$ can classify $\sim 99 \%$ of observed galaxies (Yip et al. 2004). These components thus provide a reasonable representation of the host spectrum, avoiding unphysical galaxy models which can be derived when many low-significance eigencomponents are included.

This procedure gives the best decomposition for a given SN template spectrum $S$. We can, of course, then perform this minimization for each plausible $S$ (i.e., different SN types and ages). Figure 6 shows two examples of how the FoM $\chi_{v}^{2}$ varies as $S$, type, and date change. In the upper panel of Figure 6, the large improvement near the best global maximum shows a definitive typing as an SN Ia (and a well-constrained date and decomposition). In the bottom panel of Figure 6, a standard SN Ia also provides the best fit, although an SN 1991T template is only modestly worse. Note, however, that both acceptable types show a similar well-constrained age.

If the data had Gaussian errors with the correct amplitude and the model describes the data well, we would expect $\chi_{v}^{2}$ to approach 1 , with a spread of $\Delta \chi_{v}^{2} \approx v^{-1 / 2}$ where $\Delta \chi_{v}^{2}$ stands for the difference between one solution and the best-fitted solution. In practice, however, the errors may be mis-estimated and/or the model may be wrong, giving larger $\chi_{v}^{2}$. One quantity that can be used under these conditions to distinguish between two models is a quality factor defined by

$$
Q=\frac{\Delta \chi_{v}^{2} \sqrt{v}}{\chi_{v}^{2}}
$$


Table 3

SDSS-II SN Redshift Cross-Correlation and Type Analysis

\begin{tabular}{|c|c|c|c|c|c|c|c|c|c|c|}
\hline IAUC ID $^{\mathrm{a}}$ & SNID $^{b}$ & Epoch $_{p}{ }^{c}$ & Epoch $_{\mathrm{s}}{ }^{\mathrm{d}}$ & Type $_{S N}{ }^{e}$ & $z_{\mathrm{SN}}{ }^{\mathrm{f}}$ & SN Temp $\mathrm{g}, \mathrm{h}$ & $r_{\mathrm{SN}_{\mathrm{N}}}^{\mathrm{i}}$ & $z_{g}{ }^{\mathrm{j}}$ & Type $_{\mathrm{g}}{ }^{\mathrm{k}}$ & $r_{g}{ }^{1}$ \\
\hline 2005ed & 722 & 14 & 17 & Ia & 0.087 & & 11.9 & 0.0861 & E/S0 & 17.4 \\
\hline $2005 \mathrm{ef}$ & 739 & 15 & 16 & Ia & 0.105 & & 12.9 & 0.1070 & E/S0 & 16.5 \\
\hline 2005ei & 744 & 11 & 8 & Ia & 0.123 & & 4.4 & 0.1282 & $\mathrm{Sm} / \mathrm{Im}$ & 4.7 \\
\hline 2005eg & 762 & 0 & -1 & Ia & 0.189 & & 7.5 & 0.1908 & $\mathrm{Sa}$ & 13.3 \\
\hline $2005 \mathrm{ex}$ & 774 & 14 & 16 & Ia & 0.090 & & 10.4 & 0.0933 & $\mathrm{Sa}$ & 9.5 \\
\hline 2005 ex & 774 & 22 & & Galaxy & & & & 0.0936 & $\mathrm{Sa}$ & 12.0 \\
\hline$\ldots$ & 779 & 110 & & Galaxy & & & & 0.2377 & $\mathrm{Sbc} / \mathrm{Sc}$ & 16.8 \\
\hline $2005 \mathrm{ez}$ & 1032 & 14 & 22 & Ia & 0.133 & & 8.4 & 0.1293 & $\mathrm{Sa}$ & 10.3 \\
\hline $2005 \mathrm{fg}$ & 1112 & 10 & 7 & Ia & 0.258 & & 4.3 & 0.2577 & $\mathrm{Sb}$ & 8.4 \\
\hline 2005lb & 1114 & 43 & 30 & II & 0.031 & 99em & 7.2 & 0.0245 & $\mathrm{Sm} / \mathrm{Im}$ & 9.2 \\
\hline \multirow[t]{2}{*}{$2005 \mathrm{fc}$} & 1119 & 17 & 17 & Ia & 0.298 & & 11.6 & 0.2974 & $\mathrm{Sm} / \mathrm{Im}$ & 17.0 \\
\hline & 1166 & 17 & 0 & Ia & 0.385 & & 9.6 & 0.3824 & E/S0 & 10.6 \\
\hline $2005 \mathrm{ff}$ & 1241 & 1 & 3 & Ia & 0.087 & & 21.6 & & & \\
\hline $2005 \mathrm{fd}$ & 1253 & 6 & 4 & Ia & 0.262 & & 20.6 & & & \\
\hline $2005 \mathrm{fd}$ & 1253 & 6 & & Galaxy & & & & 0.2699 & $\mathrm{Sbc/Sc}$ & 2.1 \\
\hline $2005 \mathrm{fd}$ & 1253 & 8 & 2 & Ia & 0.264 & & 8.7 & & & \\
\hline $2005 \mathrm{fe}$ & 1316 & 22 & 20 & Ia & 0.216 & & 7.2 & 0.2166 & $\mathrm{Sm} / \mathrm{Im}$ & 3.1 \\
\hline \multirow{2}{*}{$2005 \mathrm{fh}$} & 1371 & 7 & 12 & Ia & 0.130 & & 4.1 & 0.1189 & E/S0 & 8.4 \\
\hline & 1461 & 113 & & Galaxy & & & & 0.3407 & $\mathrm{E} / \mathrm{SO}$ & 8.5 \\
\hline $20051 \mathrm{c}$ & 1472 & 65 & 159 & II & 0.019 & 99em & 15.8 & 0.0129 & $\mathrm{Sm} / \mathrm{Im}$ & 7.9 \\
\hline$\ldots$ & 1525 & 99 & & Galaxy & & & & 0.1072 & $\mathrm{Sa}$ & 7.0 \\
\hline$\ldots$ & 1545 & -2 & & Galaxy & & & & 0.0987 & E/S0 & 16.4 \\
\hline $2005 \mathrm{fb}$ & 1580 & 4 & 9 & Ia & 0.184 & & 5.8 & & & \\
\hline$\ldots$ & 1595 & 11 & & Galaxy & & & & 0.2136 & E/S0 & 14.3 \\
\hline$\ldots$ & 1632 & 42 & & Galaxy & & & & 0.0575 & $\mathrm{Sm} / \mathrm{Im}$ & 25.4 \\
\hline$\ldots$ & 1686 & 11 & 41 & Ia? & 0.143 & & 8.6 & 0.1364 & $\mathrm{Sm} / \mathrm{Im}$ & 13.8 \\
\hline$\ldots$ & 1688 & 14 & 14 & Ia & 0.357 & & 17.5 & 0.3587 & $\mathrm{Sm} / \mathrm{Im}$ & 16.6 \\
\hline$\ldots$ & 1740 & 15 & & Galaxy & & & & 0.1673 & E/S0 & 20.0 \\
\hline $2005 \mathrm{fj}$ & 1794 & 15 & 15 & Ia & 0.138 & & 10.1 & 0.1426 & $\mathrm{Sm} / \mathrm{Im}$ & 3.3 \\
\hline 2005hl & 2000 & 34 & 19 & $\mathrm{Ib}$ & 0.037 & 90I & 3.0 & 0.0243 & $\mathrm{Sm} / \mathrm{Im}$ & 17.7 \\
\hline 2005 fo & 2017 & 3 & 0 & Ia & 0.254 & & 8.6 & 0.2616 & $\mathrm{Sbc/Sc}$ & 5.1 \\
\hline $2005 \mathrm{fl}$ & 2030 & 29 & 37 & Ia & 0.237 & & 2.1 & 0.2336 & $\mathrm{Sm} / \mathrm{Im}$ & 4.5 \\
\hline $2005 \mathrm{fm}$ & 2031 & 1 & 2 & Ia & 0.154 & & 10.0 & 0.1530 & $\mathrm{Sm} / \mathrm{Im}$ & 2.1 \\
\hline $2005 \mathrm{fk}$ & 2053 & 20 & 3 & Ic & 0.266 & 02ap & & 0.2643 & $\mathrm{Sm} / \mathrm{Im}$ & 4.4 \\
\hline$\ldots$ & 2063 & -4 & & Galaxy & & & & 0.1920 & $\mathrm{Sbc} / \mathrm{Sc}$ & 15.9 \\
\hline $2005 \mathrm{fn}$ & 2102 & 22 & 16 & Ia & 0.095 & & 8.2 & 0.0958 & $\mathrm{Sm} / \mathrm{Im}$ & 4.2 \\
\hline $2005 \mathrm{fr}$ & 2165 & 7 & 8 & Ia & 0.288 & & 17.7 & & & \\
\hline $2005 f y$ & 2246 & 12 & 2 & Ia & 0.193 & & 13.4 & 0.1952 & $\mathrm{Sm} / \mathrm{Im}$ & 3.7 \\
\hline 2005 fy & 2246 & 12 & 1 & Ia & 0.201 & & 6.7 & 0.1954 & $\mathrm{Sa}$ & 3.2 \\
\hline $2005 \mathrm{ey}$ & 2308 & 8 & 3 & Ia & 0.148 & & 10.6 & & & \\
\hline $2005 f p$ & 2330 & 8 & 9 & Ia & 0.208 & & 14.6 & 0.2132 & $\mathrm{Sb}$ & 9.9 \\
\hline $2005 f q$ & 2366 & 58 & 44 & II & 0.144 & $99 \mathrm{em}$ & 6.1 & 0.1454 & $\mathrm{Sb}$ & 5.3 \\
\hline$\ldots$ & 2371 & 80 & & Galaxy & & & & 0.0508 & $\mathrm{Sm} / \mathrm{Im}$ & 9.7 \\
\hline $2005 \mathrm{ft}$ & 2372 & 1 & -4 & Ia & 0.183 & & 7.7 & 0.1798 & E/S0 & 3.8 \\
\hline $2005 \mathrm{ft}$ & 2372 & 3 & 6 & Ia & 0.176 & & 6.2 & 0.1812 & $\mathrm{Sm} / \mathrm{Im}$ & 5.3 \\
\hline $2005 \mathrm{fi}$ & 2422 & 4 & 3 & Ia & 0.265 & & 11.8 & & & \\
\hline $2005 \mathrm{fu}$ & 2440 & 6 & 5 & Ia & 0.193 & & 17.5 & & & \\
\hline $2005 \mathrm{fs}$ & 2533 & 8 & 6 & Ia & 0.340 & & 4.6 & & & \\
\hline $2005 \mathrm{fv}$ & 2561 & -3 & -5 & Ia & 0.121 & & 6.4 & & & \\
\hline $2005 \mathrm{fv}$ & 2561 & -1 & -3 & Ia & 0.124 & & 5.8 & & & \\
\hline $2005 \mathrm{fv}$ & 2561 & -1 & -1 & Ia & 0.121 & & 5.7 & & & \\
\hline $2005 \mathrm{fv}$ & 2561 & 2 & & Galaxy & & & & 0.1184 & $\mathrm{Sb}$ & 5.2 \\
\hline $2005 \mathrm{fw}$ & 2635 & 31 & 27 & Ia & 0.140 & & 22.2 & 0.1433 & $\mathrm{Sbc} / \mathrm{Sc}$ & 19.0 \\
\hline $2005 \mathrm{fw}$ & 2635 & -1 & -2 & Ia & 0.144 & & 7.3 & 0.1434 & $\mathrm{Sm} / \mathrm{Im}$ & 18.8 \\
\hline$\ldots$ & 2661 & 25 & 5 & II & 0.201 & $99 \mathrm{em}$ & 4.6 & 0.1925 & $\mathrm{Sbc} / \mathrm{Sc}$ & 7.5 \\
\hline $2005 \mathrm{fa}$ & 2689 & 1 & 1 & Ia & 0.180 & & 8.3 & & & \\
\hline $2005 \mathrm{hm}$ & 2744 & 20 & 51 & $\mathrm{Ib}$ & 0.040 & $90 \mathrm{I}$ & 9.2 & 0.0350 & $\mathrm{Sm} / \mathrm{Im}$ & 5.0 \\
\hline$\ldots$ & 2746 & 18 & 2 & Ia? & & & & 0.1201 & $\mathrm{Sm} / \mathrm{Im}$ & 14.8 \\
\hline$\ldots$ & 2746 & 18 & 4 & Ia? & & & & 0.1201 & $\mathrm{Sb}$ & 15.0 \\
\hline $2005 \mathrm{fx}$ & 2789 & 10 & 2 & Ia & 0.295 & & 13.6 & 0.2903 & E/S0 & 10.6 \\
\hline$\ldots$ & 2864 & 88 & & Galaxy & & & & 0.2441 & $\mathrm{Sa}$ & 6.8 \\
\hline $2005 \mathrm{fz}$ & 2916 & -5 & 0 & Ia & 0.119 & & 14.9 & & & \\
\hline $2005 \mathrm{fz}$ & 2916 & -3 & 3 & Ia & 0.121 & & 6.5 & & & \\
\hline $2005 \mathrm{fz}$ & 2916 & 1 & 7 & Ia & 0.122 & & 20.8 & 0.1242 & $\mathrm{Sa}$ & 4.3 \\
\hline
\end{tabular}


Table 3

(Continued)

\begin{tabular}{|c|c|c|c|c|c|c|c|c|c|c|}
\hline IAUC ID $^{\mathrm{a}}$ & SNID $^{\mathrm{b}}$ & Epoch $_{p}{ }^{c}$ & Epoch $_{\mathrm{s}}{ }^{\mathrm{d}}$ & Type $_{S N}{ }^{\text {e }}$ & $z_{\mathrm{SN}}{ }^{\mathrm{f}}$ & SN Temp ${ }^{g, h}$ & $r_{\mathrm{SN}}{ }^{\mathrm{i}}$ & $z_{g}{ }^{\mathrm{j}}$ & Type $_{\mathrm{g}}{ }^{\mathrm{k}}$ & $\overline{r_{g}{ }^{1}}$ \\
\hline$\ldots$ & 2928 & 91 & & Galaxy & & & & 0.1490 & $\mathrm{Sm} / \mathrm{Im}$ & 5.3 \\
\hline$\ldots$ & 2929 & 20 & & Galaxy & & & & 0.1192 & $\mathrm{Sa}$ & 10.8 \\
\hline $2005 \mathrm{go}$ & 2943 & 13 & 14 & Ia & 0.258 & & 7.1 & 0.2654 & $\mathrm{Sm} / \mathrm{Im}$ & 4.4 \\
\hline $2005 \mathrm{gp}$ & 2992 & 12 & 11 & Ia & 0.118 & & 16.9 & 0.1268 & $\mathrm{Sb}$ & 5.9 \\
\hline $2005 \mathrm{gp}$ & 2992 & 30 & 31 & Ia & 0.128 & & 7.2 & 0.1251 & $\mathrm{Sa}$ & 6.4 \\
\hline $2005 \mathrm{ga}$ & 3080 & -5 & -10 & Ia & 0.173 & & 6.3 & 0.1750 & $\mathrm{Sa}$ & 13.4 \\
\hline $2005 \mathrm{ga}$ & 3080 & -5 & -6 & Ia & 0.171 & & 9.7 & 0.1739 & $\mathrm{Sa}$ & 8.0 \\
\hline $2005 \mathrm{ga}$ & 3080 & 24 & 23 & Ia & 0.173 & & 15.1 & 0.1742 & $\mathrm{Sb}$ & 14.0 \\
\hline $2005 \mathrm{gc}$ & 3087 & -2 & -6 & Ia & 0.156 & & 10.7 & 0.1646 & $\mathrm{Sm} / \mathrm{Im}$ & 11.3 \\
\hline $2005 \mathrm{gs}$ & 3199 & 8 & 3 & Ia & 0.266 & & 4.7 & 0.2511 & $\mathrm{Sb}$ & 5.4 \\
\hline $2005 \mathrm{gh}$ & 3241 & -6 & 6 & Ia? & 0.391 & & 2.0 & & & \\
\hline $2005 \mathrm{hn}$ & 3256 & 17 & 13 & Ia & 0.116 & & 6.8 & 0.1076 & $\mathrm{Sm} / \mathrm{Im}$ & 4.5 \\
\hline $2005 \mathrm{gd}$ & 3317 & -5 & -4 & Ia & 0.163 & & 11.6 & 0.1618 & $\mathrm{Sm} / \mathrm{Im}$ & 4.2 \\
\hline $2005 \mathrm{ge}$ & 3331 & -8 & -10 & Ia? & 0.206 & & 6.8 & & & \\
\hline $2005 \mathrm{gr}$ & 3377 & 13 & 4 & Ia & 0.243 & & 14.0 & 0.2451 & $\mathrm{Sm} / \mathrm{Im}$ & 4.0 \\
\hline $2005 \mathrm{gf}$ & 3451 & -2 & -2 & Ia & 0.246 & & & 0.2500 & $\mathrm{Sa}$ & 13.6 \\
\hline $2005 \mathrm{gg}$ & 3452 & -6 & -5 & Ia & 0.234 & & 9.1 & 0.2304 & $\mathrm{Sm} / \mathrm{Im}$ & 12.8 \\
\hline$\ldots$ & 3508 & 87 & & Galaxy & & & & 0.4967 & $\mathrm{Sm} / \mathrm{Im}$ & 8.5 \\
\hline$\ldots$ & 3508 & 92 & & Galaxy & & & & 0.4967 & $\mathrm{Sm} / \mathrm{Im}$ & 8.5 \\
\hline$\ldots$ & 3535 & 103 & & Galaxy & & & & 0.3080 & $\mathrm{Sbc} / \mathrm{Sc}$ & 5.8 \\
\hline $2005 \mathrm{gb}$ & 3592 & -5 & -5 & Ia & 0.089 & & 21.0 & 0.0863 & $\mathrm{Sb}$ & 21.9 \\
\hline $2005 \mathrm{gb}$ & 3592 & -1 & -1 & Ia & 0.087 & & 37.1 & & & \\
\hline $2005 \mathrm{gb}$ & 3592 & 11 & & Ia & & & & 0.0864 & $\mathrm{Sa}$ & 7.0 \\
\hline $2005 \mathrm{gi}$ & 3818 & 38 & 5 & II & 0.055 & $87 a$ & 4.5 & 0.0500 & $\mathrm{Sm} / \mathrm{Im}$ & 8.5 \\
\hline 2005ho & 3901 & -16 & & Ia & & & & 0.0626 & $\mathrm{Sm} / \mathrm{Im}$ & 22.1 \\
\hline 2005ho & 3901 & 6 & 5 & Ia & 0.063 & & 14.4 & 0.0636 & $\mathrm{Sm} / \mathrm{Im}$ & 4.4 \\
\hline$\ldots$ & 3959 & 103 & & Galaxy & & & & 0.1833 & $\mathrm{Sbc} / \mathrm{Sc}$ & 9.2 \\
\hline$\ldots$ & 4000 & 17 & 10 & Ia & 0.268 & & 3.8 & 0.2786 & $\mathrm{Sm} / \mathrm{Im}$ & 7.0 \\
\hline $2005 \mathrm{gt}$ & 4012 & 21 & 15 & Ic & 0.031 & $99 \mathrm{ex}$ & 4.7 & 0.0252 & $\mathrm{Sm} / \mathrm{Im}$ & 3.3 \\
\hline $2005 \mathrm{gw}$ & 4046 & 10 & 3 & Ia & 0.277 & & 8.4 & & & \\
\hline$\ldots$ & 4064 & 5 & & Galaxy & & & & 0.1559 & E/S0 & 8.3 \\
\hline$\ldots$ & 4236 & 79 & & Galaxy & & & & 0.3432 & $\mathrm{Sb}$ & 10.5 \\
\hline $2005 \mathrm{gu}$ & 4241 & 21 & 14 & Ia & 0.3320 & & 5.5 & 0.3320 & $\mathrm{Sm} / \mathrm{Im}$ & 4.2 \\
\hline$\ldots$ & 4281 & 2 & & Galaxy & & & & 0.2132 & E/S0 & 5.8 \\
\hline$\ldots$ & 4307 & 81 & & Galaxy & & & & 0.2720 & $\mathrm{Sb}$ & 3.2 \\
\hline$\ldots$ & 4311 & 77 & & Galaxy & & & & 0.2953 & $\mathrm{Sa}$ & 10.4 \\
\hline $2005 \mathrm{gj}^{\mathrm{m}}$ & 4524 & -14 & & Ia & & & & 0.0618 & $\mathrm{Sbc} / \mathrm{Sc}$ & 11.0 \\
\hline $2005 \mathrm{gj}$ & 4524 & -12 & & Ia & & & & 0.0622 & $\mathrm{Sm} / \mathrm{Im}$ & 12.8 \\
\hline $2005 \mathrm{gj}$ & 4524 & -8 & & Ia & & & & 0.0618 & $\mathrm{Sm} / \mathrm{Im}$ & 9.8 \\
\hline $2005 \mathrm{gj}$ & 4524 & -3 & 4 & Ia & 0.064 & & 2.4 & 0.0618 & $\mathrm{Sbc} / \mathrm{Sc}$ & 8.8 \\
\hline $2005 \mathrm{gj}$ & 4524 & 7 & & Ia & & & & 0.0613 & $\mathrm{Sbc} / \mathrm{Sc}$ & 16.4 \\
\hline $2005 \mathrm{gj}$ & 4524 & 10 & & Ia & & & & 0.0618 & $\mathrm{Sbc} / \mathrm{Sc}$ & 14.4 \\
\hline $2005 \mathrm{gj}$ & 4524 & 18 & & Ia & & & & 0.0616 & $\mathrm{Sbc} / \mathrm{Sc}$ & 12.3 \\
\hline $2005 \mathrm{gj}$ & 4524 & 26 & & Ia & & & & & & \\
\hline $2005 \mathrm{gj}$ & 4524 & 28 & & Ia & & & & & & \\
\hline $2005 \mathrm{gj}$ & 4524 & 28 & & Ia & & & & 0.0620 & $\mathrm{Sbc} / \mathrm{Sc}$ & 10.8 \\
\hline $2005 \mathrm{gj}$ & 4524 & 42 & & Ia & & & & 0.0619 & $\mathrm{Sbc} / \mathrm{Sc}$ & 11.5 \\
\hline $2005 \mathrm{gj}$ & 4524 & 44 & 42 & Ia & 0.067 & & 7.2 & 0.0616 & $\mathrm{Sbc} / \mathrm{Sc}$ & 12.2 \\
\hline $2005 \mathrm{gj}$ & 4524 & 54 & 42 & Ia & 0.068 & & 8.1 & 0.0615 & $\mathrm{Sbc} / \mathrm{Sc}$ & 11.3 \\
\hline $2005 \mathrm{gj}$ & 4524 & 71 & 45 & Ia & 0.070 & & 6.0 & 0.0616 & $\mathrm{Sbc} / \mathrm{Sc}$ & 17.0 \\
\hline $2005 \mathrm{gv}$ & 4577 & 9 & 1 & Ia? & 0.363 & & & & & \\
\hline$\ldots$ & 4676 & 77 & & Galaxy & & & & 0.2446 & $\mathrm{Sa}$ & 6.6 \\
\hline $2005 \mathrm{gy}$ & 4679 & 2 & -2 & Ia & 0.338 & & 3.1 & 0.3324 & $\mathrm{Sm} / \mathrm{Im}$ & 4.4 \\
\hline $2005 \mathrm{gx}$ & 5103 & -5 & 7 & Ia & 0.146 & & 4.9 & & & \\
\hline $2005 \mathrm{gq}$ & 5183 & 13 & -4 & Ia & 0.382 & & 3.5 & 0.3898 & $\mathrm{Sb}$ & 5.6 \\
\hline $2005 \mathrm{hp}$ & 5350 & 8 & 3 & Ia & 0.174 & & 6.1 & 0.1754 & $\mathrm{Sbc} / \mathrm{Sc}$ & 13.5 \\
\hline$\ldots$ & 5378 & 76 & & Galaxy & & & & 0.2511 & E/S0 & 3.4 \\
\hline $2005 \mathrm{hs}$ & 5391 & 13 & 14 & Ia & 0.295 & & 7.5 & 0.3009 & $\mathrm{Sm} / \mathrm{Im}$ & 4.2 \\
\hline $2005 \mathrm{hr}$ & 5395 & 4 & 1 & Ia & 0.115 & & 4.4 & 0.1170 & $\mathrm{Sbc} / \mathrm{Sc}$ & 5.5 \\
\hline 2005hu & 5533 & 6 & 5 & Ia & 0.224 & & 37.3 & 0.2197 & $\mathrm{Sm} / \mathrm{Im}$ & 28.4 \\
\hline $2005 \mathrm{hx}$ & 5549 & 0 & 1 & Ia & 0.121 & & 12.9 & & & \\
\hline 2005hy & 5550 & -3 & -1 & Ia & 0.154 & & 5.0 & 0.1562 & $\mathrm{Sb}$ & 7.6 \\
\hline $2005 \mathrm{hw}$ & 5588 & 10 & 8 & Ia & 0.407 & & 3.1 & 0.4102 & $\mathrm{E} / \mathrm{S} 0$ & 8.2 \\
\hline $2005 \mathrm{hv}$ & 5635 & 5 & 3 & Ia & 0.181 & & 10.4 & 0.1795 & $\mathrm{Sm} / \mathrm{Im}$ & 4.1 \\
\hline
\end{tabular}


Table 3

(Continued)

\begin{tabular}{|c|c|c|c|c|c|c|c|c|c|c|}
\hline IAUC ID $^{\mathrm{a}}$ & SNID $^{\mathrm{b}}$ & Epoch $_{p}{ }^{c}$ & Epoch $_{\mathrm{s}}{ }^{\mathrm{d}}$ & Type $_{S N}{ }^{e}$ & $z_{\mathrm{SN}}{ }^{\mathrm{f}}$ & SN Temp $p^{g, h}$ & $r_{\mathrm{SN}}^{\mathrm{i}}$ & $z g^{\mathrm{j}}$ & Type $_{\mathrm{g}}{ }^{\mathrm{k}}$ & $r_{g}{ }^{1}$ \\
\hline$\ldots$ & 5673 & 11 & & Galaxy & & & & 0.3793 & E/S0 & 12.1 \\
\hline $2005 \mathrm{ia}$ & 5717 & 9 & 3 & Ia & 0.251 & & 15.9 & 0.2517 & $\mathrm{Sm} / \mathrm{Im}$ & 6.7 \\
\hline $2005 \mathrm{jz}$ & 5736 & 17 & 14 & Ia & 0.253 & & 8.0 & & & \\
\hline $2005 i b$ & 5737 & 9 & 3 & $\mathrm{Ia}$ & 0.393 & & 18.2 & 0.3930 & $\mathrm{Sm} / \mathrm{Im}$ & 8.8 \\
\hline $2005 \mathrm{hz}$ & 5751 & 2 & 0 & Ia & 0.133 & & 3.9 & 0.1301 & $\mathrm{Sbc} / \mathrm{Sc}$ & 4.2 \\
\hline $2005 \mathrm{hz}$ & 5751 & 36 & 35 & Ia & 0.133 & & 7.0 & 0.1300 & $\mathrm{Sb}$ & 6.8 \\
\hline $2005 \mathrm{hq}$ & 5821 & 16 & 18 & Ia? & 0.423 & & 2.5 & & & \\
\hline $2005 \mathrm{hq}$ & 5821 & 18 & 14 & Ia? & 0.423 & & 2.0 & 0.4008 & $\mathrm{Sbc} / \mathrm{Sc}$ & 3.8 \\
\hline $2005 i c$ & 5844 & 15 & 2 & Ia & 0.314 & & 13.1 & 0.3108 & $\mathrm{Sm} / \mathrm{Im}$ & 4.1 \\
\hline 2005is & 5916 & 7 & 4 & Ia & 0.177 & & 2.4 & 0.1724 & $\mathrm{Sb}$ & 6.5 \\
\hline 2005is & 5916 & 12 & 13 & Ia & 0.177 & & 6.2 & 0.1740 & $\mathrm{Sb}$ & 5.3 \\
\hline $2005 \mathrm{hc}$ & 5944 & -3 & -4 & Ia & 0.045 & & 19.3 & & & \\
\hline $2005 \mathrm{hc}$ & 5944 & -1 & 1 & Ia & 0.044 & & 22.9 & & & \\
\hline $2005 \mathrm{hc}$ & 5944 & 34 & 34 & Ia & 0.046 & & 16.1 & 0.0453 & E/S0 & 6.8 \\
\hline $2005 \mathrm{ie}$ & 5957 & 6 & 4 & Ia & 0.286 & & 14.9 & 0.2796 & $\mathrm{Sm} / \mathrm{Im}$ & 15.0 \\
\hline$\ldots$ & 5963 & 64 & & Galaxy & & & & 0.2356 & $\mathrm{Sbc} / \mathrm{Sc}$ & 11.5 \\
\hline 2005it & 5966 & 19 & 17 & Ia & 0.301 & & 3.3 & 0.3082 & $\mathrm{Sm} / \mathrm{Im}$ & 9.0 \\
\hline$\ldots$ & 5993 & 78 & & Galaxy & & & & 0.3767 & $\mathrm{Sa}$ & 11.5 \\
\hline $2005 \mathrm{ht}$ & 5994 & 4 & 1 & Ia & 0.187 & & 11.5 & & & \\
\hline 2005if & 6057 & 3 & 7 & Ia & 0.067 & & 1.7 & 0.0675 & $\mathrm{Sm} / \mathrm{Im}$ & 5.4 \\
\hline 2005if & 6057 & 37 & 35 & Ia & 0.070 & & 9.2 & 0.0664 & $\mathrm{Sm} / \mathrm{Im}$ & 11.5 \\
\hline $2005 \mathrm{ka}$ & 6100 & 21 & 13 & Ia & 0.316 & & 4.5 & 0.3177 & $\mathrm{Sm} / \mathrm{Im}$ & 9.2 \\
\hline 2005ih & 6108 & 0 & 2 & Ia & 0.262 & & 11.5 & 0.2595 & $\mathrm{Sm} / \mathrm{Im}$ & 4.4 \\
\hline 2005iw & 6127 & 9 & 2 & Ia & 0.280 & & 5.1 & & & \\
\hline $2005 \mathrm{iv}$ & 6137 & 11 & -1 & Ia & 0.311 & & 3.5 & 0.3001 & $\mathrm{Sbc} / \mathrm{Sc}$ & 4.0 \\
\hline 2005jy & 6192 & 10 & 6 & Ia & 0.272 & & 18.2 & 0.2720 & $\mathrm{Sm} / \mathrm{Im}$ & 12.5 \\
\hline 2005ig & 6196 & 7 & 1 & Ia & 0.279 & & 11.8 & 0.2807 & E/S0 & 9.9 \\
\hline $2005 \mathrm{ii}$ & 6249 & 0 & -4 & Ia & 0.290 & & 13.7 & 0.2944 & $\mathrm{Sm} / \mathrm{Im}$ & 11.0 \\
\hline $2005 \mathrm{js}$ & 6295 & 17 & 18 & Iapec & 0.082 & $1991 \mathrm{bg}$ & 8.5 & 0.0791 & E/S0 & 12.5 \\
\hline 2005jk & 6304 & 10 & & Galaxy & & & & 0.1903 & $\mathrm{Sm} / \mathrm{Im}$ & 7.7 \\
\hline 2005jk & 6304 & 9 & 10 & Ia & 0.183 & & 23.0 & & & \\
\hline $2005 i x$ & 6315 & 1 & -5 & Ia & 0.263 & & 6.0 & 0.2670 & $\mathrm{Sm} / \mathrm{Im}$ & 5.1 \\
\hline $2005 i x$ & 6315 & 5 & 2 & Ia & 0.255 & & 15.6 & & & \\
\hline $2005 \mathrm{ij}$ & 6406 & -6 & -9 & Ia & 0.122 & & 3.6 & 0.1231 & $\mathrm{Sb}$ & 3.4 \\
\hline $2005 \mathrm{ij}$ & 6406 & 27 & 25 & Ia & 0.123 & & 17.7 & 0.1227 & $\mathrm{Sb}$ & 3.3 \\
\hline 2005id & 6422 & 1 & 2 & Ia & 0.184 & & 7.9 & & & \\
\hline$\ldots$ & 6471 & 3 & 16 & II & 0.210 & $99 \mathrm{em}$ & 5.8 & 0.2016 & $\mathrm{Sbc} / \mathrm{Sc}$ & 5.2 \\
\hline $2005 \mathrm{hj}^{\mathrm{n}}$ & 6558 & & & Ia & & & & & & \\
\hline 2005jd & 6649 & 4 & 6 & Ia & 0.314 & & 3.8 & & & \\
\hline $2005 \mathrm{jd}$ & 6649 & 0 & -5 & Ia & 0.330 & & 3.8 & & & \\
\hline & 6696 & 5 & & Ia? & & & & 0.2379 & $\mathrm{Sm} / \mathrm{Im}$ & 8.5 \\
\hline $2005 \mathrm{ik}$ & 6699 & -1 & 2 & Ia & 0.312 & & 14.0 & 0.3106 & $\mathrm{Sb}$ & 8.8 \\
\hline $2005 \mathrm{ik}$ & 6699 & 7 & 2 & Ia & 0.313 & & 19.5 & & & \\
\hline$\ldots$ & 6714 & 54 & & Galaxy & & & & 0.4137 & $\mathrm{Sm} / \mathrm{Im}$ & 12.3 \\
\hline 2005iu & 6773 & -5 & -4 & Ia & 0.088 & & 6.2 & 0.0904 & $\mathrm{Sa}$ & 5.8 \\
\hline 2005iy & 6777 & 17 & 2 & Ia & 0.404 & & 3.0 & 0.4043 & $\mathrm{Sb}$ & 2.9 \\
\hline 2005iy & 6777 & 20 & 8 & Ia & 0.394 & & 2.0 & & & \\
\hline $2005 \mathrm{iz}$ & 6780 & 12 & 13 & Ia & 0.202 & & 8.0 & & & \\
\hline 2005jf & 6852 & -3 & & Ia? & & & & 0.3006 & $\mathrm{Sa}$ & 8.6 \\
\hline $2005 \mathrm{ja}$ & 6924 & 5 & 0 & Ia & 0.328 & & 8.2 & & & \\
\hline $2005 \mathrm{jc}$ & 6933 & -1 & 0 & Ia & 0.214 & & 6.7 & & & \\
\hline $2005 \mathrm{jc}$ & 6933 & 2 & 1 & Ia & 0.213 & & 15.8 & & & \\
\hline $2005 \mathrm{jl}$ & 6936 & 0 & 0 & Ia & 0.186 & & 19.8 & 0.1810 & $\mathrm{Sm} / \mathrm{Im}$ & 3.3 \\
\hline $2005 \mathrm{je}$ & 6962 & 13 & 13 & Ia? & 0.097 & & 2.1 & 0.0930 & $\mathrm{Sa}$ & 7.5 \\
\hline$\ldots$ & 6968 & 12 & 13 & Ia? & 0.119 & & 2.8 & 0.0980 & $\mathrm{Sb}$ & 7.9 \\
\hline$\ldots$ & 7017 & 318 & 405 & Ia & 0.271 & $2005 \mathrm{gj}$ & 13.8 & 0.2684 & $\mathrm{Sm} / \mathrm{Im}$ & 15.7 \\
\hline$\ldots$ & 7099 & 0 & & Galaxy & & & & 0.2186 & $\mathrm{Sa}$ & 5.4 \\
\hline$\ldots$ & 7102 & 7 & & Galaxy & & & & 0.1965 & $\mathrm{Sm} / \mathrm{Im}$ & 3.2 \\
\hline 2005jg & 7143 & 1 & -1 & Ia & 0.304 & & 6.6 & & & \\
\hline 2005jh & 7147 & 2 & 2 & Ia & 0.109 & & 12.1 & 0.1099 & E/S0 & 4.7 \\
\hline 2005jh & 7147 & -1 & 1 & Ia & 0.109 & & 6.2 & 0.1100 & E/S0 & 4.0 \\
\hline 2005jm & 7243 & -5 & -4 & Ia & 0.197 & & 23.7 & 0.2037 & $\mathrm{Sm} / \mathrm{Im}$ & 10.1 \\
\hline $2005 \mathrm{kn}$ & 7335 & 8 & 14 & Ia? & 0.197 & & 1.4 & & & \\
\hline 2005jb & 7426 & -7 & 0 & Ia? & 0.384 & & 2.2 & & & \\
\hline
\end{tabular}


Table 3

(Continued)

\begin{tabular}{|c|c|c|c|c|c|c|c|c|c|c|}
\hline IAUC ID $^{\mathrm{a}}$ & SNID $^{\mathrm{b}}$ & Epoch $_{p}{ }^{c}$ & Epoch $_{\mathrm{s}}{ }^{\mathrm{d}}$ & Type $_{S N}{ }^{e}$ & $z_{\mathrm{SN}}{ }^{\mathrm{f}}$ & SN Temp, & $r_{\mathrm{SN}}^{\mathrm{i}}$ & $z g^{\mathrm{j}}$ & Type $_{\mathrm{g}}{ }^{\mathrm{k}}$ & $r_{g}{ }^{1}$ \\
\hline$\ldots$ & 7444 & 44 & & Galaxy & & & & 0.2499 & $\mathrm{Sb}$ & 6.7 \\
\hline $2005 \mathrm{jx}$ & 7460 & 7 & 8 & Ia? & 0.218 & & 2.1 & 0.2103 & $\mathrm{Sb}$ & 3.3 \\
\hline $2005 \mathrm{ji}$ & 7473 & 1 & 2 & Ia & 0.221 & & 5.1 & & & \\
\hline $2005 \mathrm{ji}$ & 7473 & 2 & 2 & Ia & 0.216 & & 18.3 & & & \\
\hline 2005jn & 7475 & 5 & 2 & Ia & 0.322 & & 8.5 & & & \\
\hline 2005jn & 7475 & 5 & 6 & Ia & 0.326 & & 5.7 & & & \\
\hline 2005jo & 7512 & -1 & 2 & Ia & 0.219 & & 11.6 & & & \\
\hline$\ldots$ & 7647 & 49 & & Galaxy & & & & 0.3854 & $\mathrm{Sm} / \mathrm{Im}$ & 11.0 \\
\hline $2005 \mathrm{jw}$ & 7779 & 8 & 7 & Ia & 0.374 & & 4.2 & 0.3812 & $\mathrm{Sbc} / \mathrm{Sc}$ & 3.0 \\
\hline 2005jp & 7847 & 0 & -3 & Ia & 0.209 & & 10.9 & 0.2124 & $\mathrm{Sb}$ & 3.2 \\
\hline $2005 \mathrm{ir}$ & 7876 & -9 & -5 & Ia & 0.062 & & 8.3 & 0.0762 & $\mathrm{Sb}$ & 24.7 \\
\hline \multirow[t]{2}{*}{2005 ir } & 7876 & -5 & -3 & Ia & 0.070 & & 7.4 & 0.0761 & $\mathrm{Sbc} / \mathrm{Sc}$ & 16.1 \\
\hline & 7922 & 0 & & Galaxy & & & & 0.1822 & $\mathrm{Sb}$ & 16.9 \\
\hline $2005 \mathrm{jj}$ & 7947 & 7 & 6 & Ia? & 0.368 & & 6.1 & & & \\
\hline $2005 \mathrm{jv}$ & 8030 & 8 & 6 & Ia & 0.422 & & 5.7 & & & \\
\hline 2005ju & 8046 & -2 & -4 & Ia & 0.251 & & 9.4 & 0.2593 & $\mathrm{E} / \mathrm{S} 0$ & 11.6 \\
\hline $2005 \mathrm{hk}^{\mathrm{o}}$ & 8151 & -7 & & Ia & & & & & & \\
\hline 2005hk & 8151 & -6 & & Ia & & & & 0.0124 & $\mathrm{Sm} / \mathrm{Im}$ & 21.8 \\
\hline $2005 \mathrm{hk}$ & 8151 & -5 & & Ia & & & & 0.0125 & $\mathrm{Sm} / \mathrm{Im}$ & 3.8 \\
\hline $2005 \mathrm{hk}$ & 8151 & -1 & & Ia & & & & & & \\
\hline 2005hk & 8151 & 1 & & Ia & & & & & & \\
\hline 2005hk & 8151 & -7 & & Ia & & & & & & \\
\hline $2005 \mathrm{hk}$ & 8151 & 3 & & Ia & & & & & & \\
\hline 2005hk & 8151 & 26 & & Ia & & & & & & \\
\hline 2005ko & 8213 & 13 & 18 & Ia & 0.184 & & 9.3 & 0.1847 & $\mathrm{Sbc/Sc}$ & 3.5 \\
\hline $2005 \mathrm{mi}$ & 8495 & 23 & 22 & Ia & 0.212 & & 4.6 & 0.2144 & $\mathrm{Sb}$ & 10.1 \\
\hline 2005jt & 8598 & 5 & 20 & Ia & 0.356 & & 3.3 & 0.3606 & $\mathrm{Sm} / \mathrm{Im}$ & 7.7 \\
\hline 2005jr & 8679 & -3 & & II & & & & 0.2944 & $\mathrm{Sm} / \mathrm{Im}$ & 6.0 \\
\hline $2005 \mathrm{mh}$ & 8707 & 28 & 16 & Ia? & 0.400 & & 3.5 & 0.3951 & $\mathrm{Sb}$ & 3.2 \\
\hline \multirow{2}{*}{$2005 \mathrm{kp}$} & 8719 & -6 & -6 & Ia & 0.113 & & 9.1 & 0.1163 & $\mathrm{Sm} / \mathrm{Im}$ & 10.6 \\
\hline & 8742 & 49 & & Galaxy & & & & 0.2141 & $\mathrm{Sm} / \mathrm{Im}$ & 17.2 \\
\hline 2005ld & 8921 & 6 & 6 & Ia & 0.149 & & 6.9 & 0.1454 & $\mathrm{Sm} / \mathrm{Im}$ & 5.6 \\
\hline 2005le & 9032 & 14 & 14 & Ia & 0.253 & & 14.8 & 0.2540 & $\mathrm{Sm} / \mathrm{Im}$ & 14.3 \\
\hline $2005 \mathrm{kq}$ & 9045 & 15 & 15 & Ia & 0.393 & & 3.7 & 0.3895 & $\mathrm{Sm} / \mathrm{Im}$ & 10.0 \\
\hline $2005 \mathrm{kr}$ & 9118 & 7 & 3 & Ic & 0.124 & 02ap & 1.5 & 0.1338 & $\mathrm{Sm} / \mathrm{Im}$ & 13.1 \\
\hline 2005lg & 9207 & 15 & 15 & Ia & 0.345 & & 23.0 & 0.3500 & $\mathrm{Sb}$ & 7.1 \\
\hline 2005ks & 9273 & 8 & 19 & Ic & 0.102 & 02ap & 2.8 & 0.0989 & $\mathrm{Sm} / \mathrm{Im}$ & 23.5 \\
\hline 2005li & 9457 & 11 & 14 & Ia & 0.254 & & 4.5 & 0.2569 & $\mathrm{Sa}$ & 5.4 \\
\hline \multirow[t]{2}{*}{ 2005lh } & 9467 & 18 & 16 & Ia & 0.218 & & 8.3 & 0.2184 & $\mathrm{Sa}$ & 8.8 \\
\hline & 9954 & 40 & & Galaxy & & & & 0.2277 & $\mathrm{Sm} / \mathrm{Im}$ & 7.4 \\
\hline \multirow[t]{2}{*}{$2005 \mathrm{kt}$} & 10028 & 26 & 1 & Ia & 0.063 & & 5.9 & 0.0662 & E/S0 & 5.9 \\
\hline & 10045 & 11 & & Galaxy & & & & 0.1147 & $\mathrm{Sb}$ & 9.2 \\
\hline \multirow{2}{*}{$\begin{array}{l}2005 \mathrm{lj} \\
\ldots\end{array}$} & 10096 & 7 & 7 & Ia & 0.075 & & 4.5 & 0.0774 & $\mathrm{Sbc} / \mathrm{Sc}$ & 16.1 \\
\hline & 10106 & 39 & 37 & Ia? & 0.155 & & 3.5 & 0.1472 & $\mathrm{Sb}$ & 6.0 \\
\hline $2005 \mathrm{mk}$ & 10297 & 16 & 10 & II & 0.151 & $99 \mathrm{em}$ & 6.1 & 0.1477 & $\mathrm{Sb}$ & 4.7 \\
\hline $2005 \mathrm{~kb}$ & 10367 & 42 & 7 & II & 0.015 & $99 \mathrm{em}$ & 19.8 & 0.0155 & $\mathrm{Sbc} / \mathrm{Sc}$ & 3.2 \\
\hline $2005 \mathrm{lk}$ & 10434 & 1 & 2 & Ia & 0.109 & & 10.1 & 0.1040 & E/S0 & 10.6 \\
\hline 200511 & 10449 & 6 & 2 & Ia & 0.244 & & 13.1 & & & \\
\hline 2005lf & 10550 & 13 & 11 & Ia & 0.302 & & 13.0 & 0.3001 & $\mathrm{Sm} / \mathrm{Im}$ & 26.0 \\
\hline $2005 \mathrm{ku}$ & 10805 & -2 & 1 & Ia & 0.039 & & 17.2 & 0.0456 & $\mathrm{Sm} / \mathrm{Im}$ & 11.1 \\
\hline 20051m & 10907 & 4 & 10 & II & 0.090 & 99em & 4.4 & 0.0842 & $\mathrm{Sm} / \mathrm{Im}$ & 18.7 \\
\hline \multirow[t]{3}{*}{$\ldots$} & 10915 & 42 & & Galaxy & & & & 0.2244 & $\mathrm{Sm} / \mathrm{Im}$ & 3.9 \\
\hline & 10928 & 9 & & Galaxy & & & & 0.0815 & $\mathrm{Sbc} / \mathrm{Sc}$ & 22.3 \\
\hline & 10976 & 6 & & Galaxy & & & & 0.0971 & $\mathrm{Sa}$ & 4.6 \\
\hline $2005 \mathrm{mj}$ & 11017 & 3 & & II & & & & & & \\
\hline $2005 \mathrm{ml}$ & 11067 & 7 & 8 & Ia & 0.114 & & 5.6 & & & \\
\hline $2005 \mathrm{~mm}$ & 11206 & 19 & 7 & Ia & 0.382 & & 5.4 & 0.3819 & $\mathrm{Sa}$ & 5.2 \\
\hline 2005ln & 11300 & -1 & 0 & Ia & 0.128 & & 9.2 & 0.1370 & $\mathrm{Sm} / \mathrm{Im}$ & 4.6 \\
\hline 2005mo & 11320 & 22 & 13 & Ia & 0.278 & & 1.3 & 0.2743 & $\mathrm{Sm} / \mathrm{Im}$ & 5.5 \\
\hline $2005 \mathrm{mn}$ & 11332 & 2 & 19 & $\mathrm{Ib}$ & 0.054 & 90I & 5.8 & 0.0469 & $\mathrm{Sb}$ & 10.1 \\
\hline $2005 \mathrm{mn}$ & 11332 & 6 & 19 & $\mathrm{Ib}$ & 0.051 & 90I & 7.8 & 0.0469 & $\mathrm{Sm} / \mathrm{Im}$ & 10.0 \\
\hline $2005 \mathrm{mn}$ & 11332 & 8 & 19 & $\mathrm{Ib}$ & 0.054 & 90I & 12.6 & 0.0473 & $\mathrm{Sb}$ & 11.1 \\
\hline $2005 \mathrm{mn}$ & 11332 & 64 & 39 & $\mathrm{Ib}$ & 0.058 & 90I & 4.5 & 0.0480 & $\mathrm{Sb}$ & 3.8 \\
\hline 2005lo & 11452 & 16 & 8 & Ia & 0.299 & & 3.6 & & & \\
\hline
\end{tabular}


Table 3

(Continued)

\begin{tabular}{|c|c|c|c|c|c|c|c|c|c|c|}
\hline IAUC ID $^{\mathrm{a}}$ & SNID $^{\mathrm{b}}$ & Epoch $_{p}{ }^{c}$ & Epoch $_{\mathrm{s}}^{\mathrm{d}}$ & Type $_{\text {SN }}{ }^{\mathrm{e}}$ & $z_{\mathrm{SN}}{ }^{\mathrm{f}}$ & SN Temp $p^{g, h}$ & $r_{\mathrm{SN}}^{\mathrm{i}}$ & $z_{g}{ }^{\mathrm{j}}$ & Type $_{\mathrm{g}}{ }^{\mathrm{k}}$ & $r_{g}{ }^{1}$ \\
\hline 2005lq & 11557 & -1 & -2 & Ia & 0.380 & & 5.6 & & & \\
\hline $2005 \mathrm{mp}$ & 11650 & -1 & -1 & Ia & 0.273 & & 10.9 & & & \\
\hline 2005lp & 11864 & 16 & 3 & Ia & 0.303 & & 5.2 & & & \\
\hline $2005 \mathrm{mq}$ & 12136 & 7 & 7 & Ia & 0.350 & & 5.9 & & & \\
\hline
\end{tabular}

Notes.

${ }^{\text {a }}$ Official IAU name, when assigned.

${ }^{\mathrm{b}}$ SDSS Internal SN ID.

${ }^{\mathrm{c}}$ Spectrum day (in observer frame) relative to epoch of maximum brightness from the photometric typing.

${ }^{\mathrm{d}}$ Spectrum day (in observer frame) relative to epoch of maximum brightness from the spectroscopic cross-correlation analysis.

e Best-fitted type of the SNe. "Ia?" indicates a best match to an SN Ia template, although some features are not detected. "II?" indicates a general match to the SN II template, although expected SN II features are not all detected. "Ia-pec" indicates peculiar SN Ia.

${ }^{\mathrm{f}}$ Redshift measured from cross-correlation against SN templates. This value has been corrected for the systematic offset 0.003 . The expected redshift error is 0.005 .

$\mathrm{g}$ Template spectrum providing the best match to the observed spectrum. If blank, then the best-fitted template belongs to the Nugent SN Ia set.

${ }^{\text {h }}$ SN templates from Pun et al. (1995), Turatto et al. (1996), Hamuy et al. (2002), Gal-Yam et al. (2002), Leonard et al. (2002), Elmhamdi et al. (2004), and Nugent et al. (2002).

${ }^{i} r$ value of the rvsao.xcsao comparison with SN templates. $r_{\mathrm{SN}}>3$ is a criterion for a "good" fit. See text for details.

${ }^{\mathrm{j}}$ Redshift from cross-correlation against galaxy templates. The expected redshift error is 0.0005 .

${ }^{\mathrm{k}}$ Best-fit type of the host galaxy.

${ }^{1} r$ value for rvsao.xcsao correlation against host-galaxy templates. $r_{g}>3$ is a criterion for a "good" fit. See text for details.

${ }^{m}$ Initially identified as a normal SN Ia by cross-correlation analysis. Later, determined to be a peculiar SN Ia (SN 2002ic-like) based on detailed spectroscopic analysis.

${ }^{\mathrm{n}}$ This SN was confirmed by another group using the HET (CBET 266; Quimby et al. 2007). The spectrum is not available.

o Initially identified as a normal SN Ia by cross-correlation analysis. Later, determined to be a peculiar SN Ia (SN 1991T-like), based on detailed spectroscopic analysis.
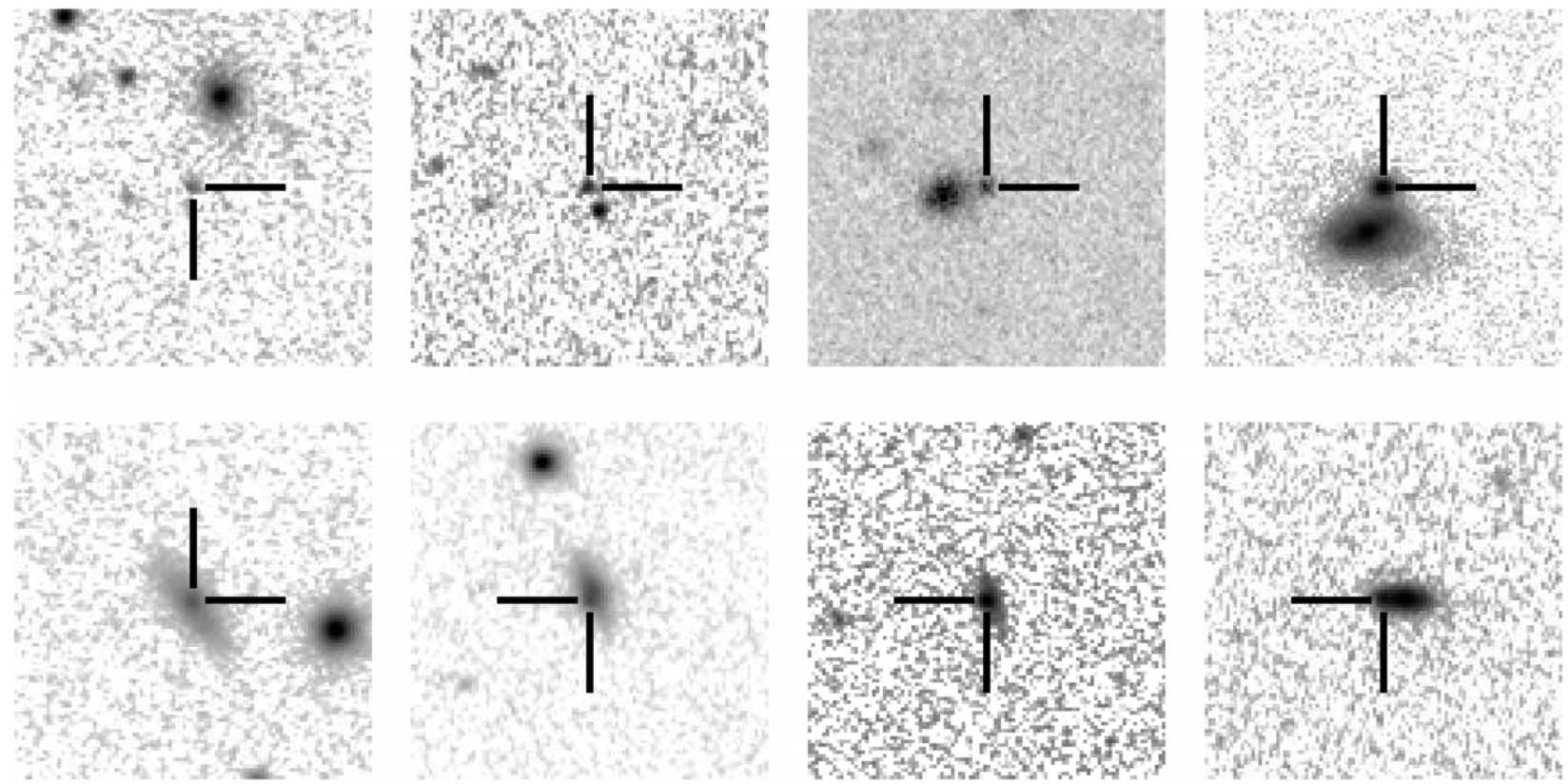

Figure 5. This panel shows images of "clean" (top row) and "heavily contaminated" (bottom row) SNe. The tick marks indicate the location of the SN.

where the $\chi_{v}^{2}$ value for the best-fitted model is inserted in the denominator to compensate for incorrect error estimates. Large $\Delta \chi_{v}^{2}$ yields large $Q$ values and thus more significant discrimination between the models. In fact, even when the models are not correct or equivalently the data suffer uncorrected systematic bias, a large value of $Q(\gtrsim 1)$ can indicate a significant discrimination between two models.
Unfortunately, we find that we do not always satisfy this criterion $(Q \gtrsim 1)$, even when visual inspection indicates a clear-type assignment, so our PCA analysis does not always definitively type the SN. While it does indeed confirm the clear SN identifications, and while the "best" type (albeit at small $\left.\Delta \chi_{v}^{2} / \chi_{v}^{2}\right)$ is often that selected by hand, there are some disagreements with the types selected manually from the $r$ 

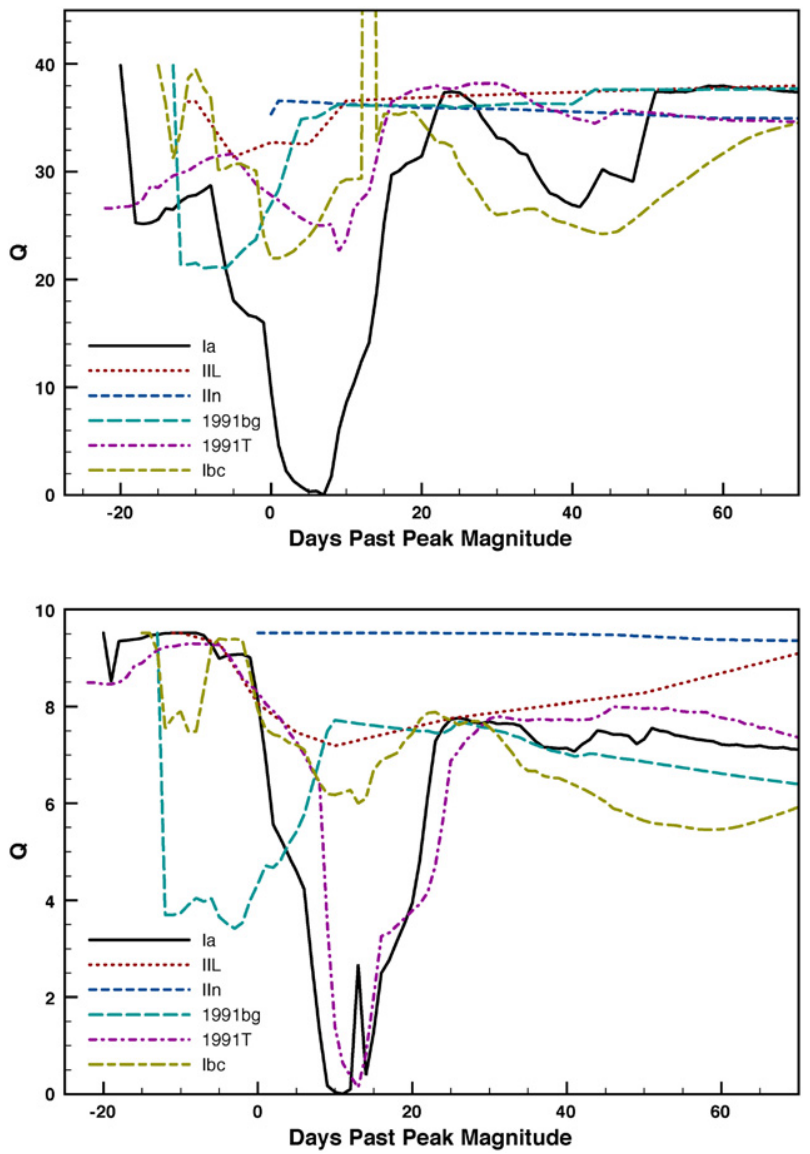

Figure 6. Two panels showing $\chi_{v}^{2}$ fitting results to various SN templates as a function of age. The object in the upper panel, with $v=943$ degrees of freedom, is classified as an SN Ia at high confidence, since the quality factor $Q=\Delta \chi_{v}^{2} \sqrt{v} / \chi_{v}^{2}$ of the next-best-type solution is $\gg 1$. The lower panel, with $v=918$ degrees of freedom, has a preferred but not definitive SN Ia classification, with $Q \ll 1$. The photometric ages for these two objects are $5 \mathrm{~d}$ and $16 \mathrm{~d}$ after $B$-band maximum respectively; the spectroscopic age estimates are consistent with this value.

(A color version of this figure is available in the online journal)

values of the cross-correlation analysis. At least for our typical data quality, it seems that PCA analysis does not provide a reliable automatic classifier for low-S/N spectra. In particular, the $\Delta \chi_{v}^{2} / \chi_{v}^{2}$ analysis has difficulty in distinguishing between $\mathrm{SN}$ Ia subtypes. Thus, in ambiguous cases, we defer to the typing from the cross-correlation analysis. However, even in these cases, the PCA analysis is valuable, as it provides a quantitative estimate of the galaxy/SN decomposition.

As noted above, we can, in principle, independently measure the absorption from the spectrum reddening. However, when we allow $c$, the coefficient that characterizes the amount of extinction and reddening, to range freely, the value at $\chi_{v}^{2}$ minimum is often higher than that determined from the lightcurve analysis. One difference is that the light-curve analysis includes an exponential prior (favoring low $A_{V}$ values), while we do not impose a prior. However, we believe that the limited accuracy of our relative spectrophotometry prevents us from making useful reddening measurements. This is seen in the large dispersion of the fit values. Also it may be problematic that our assumed extinction law, which corresponds to the standard Galactic $R_{V}=3.1$, may not apply to SNe Ia host environments. Indeed widely values have been estimated for $R_{V}$ (e.g. Branch

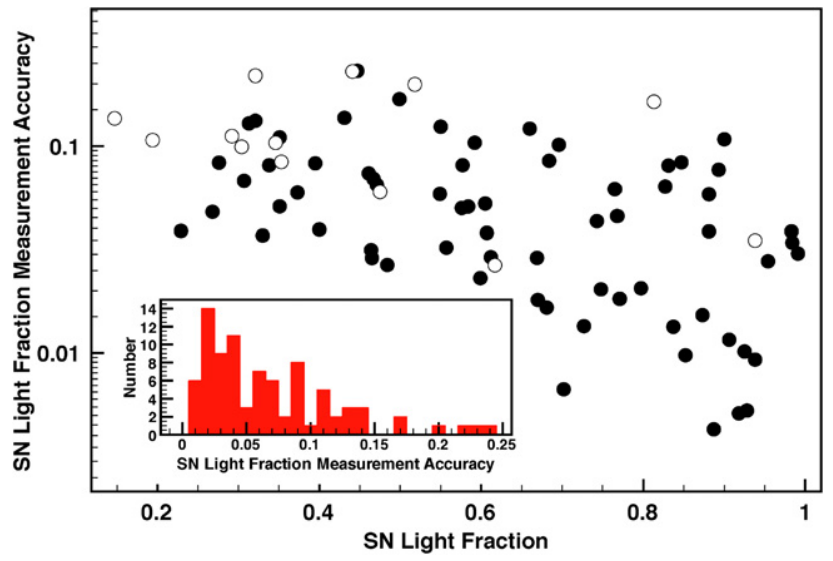

Figure 7. The accuracy of the SN component measurement (defined as the statistical error of SN light flux divided by the fraction of SN light flux) plotted versus SN fraction. "Type Ia?" (empty circles) tend to have relatively low accuracy. In most cases, the SN fraction is measured to within $<10 \%$.

(A color version of this figure is available in the online journal)

\& Tammann 1992; 1.55, Krisciunas et al. 2006; 2.5, Knop et al. 2003, Altavilla et al. 2004, etc). Accordingly, for our final decomposition we fix the value of the extinction to that obtained from the light-curve analysis. The redshift and spectral identification are not very sensitive to the value of $A_{V}$ to within their uncertainties.

Thus, adopting the $z$ and SN type from the cross-correlation analysis and the extinction from the light-curve fitting, we can use the PCA decomposition to obtain a model galaxy spectrum and a fraction of SN light together with statistical errors (twosided, asymmetric error bars with a $1 \sigma$ confidence level). The accuracy of such measurement versus the magnitude of the fraction is shown in Figure 7. The relatively low-significance points include SNe of the "Ia?" class, for which the characteristic lines were not measured with high significance, but the general spectral shape was best fitted by an SN Ia template.

In general, the typing from cross-correlation analysis and PCA decomposition were consistent. The epochs determined from both analysis mostly agree within $\sim 5$ days and are close to those estimated from photometry analysis (see Figure 8). Approximately $80 \%$ of the galaxy types determined from the PCA analyses are consistent with the cross-correlation results. No prior is assumed except for the physical constraints on the parameters ( $a>0$, constraints on the $b_{0}, b_{1}$, and $b_{2}$ to make sure the constructed galaxy type is physically plausible). The fit values are listed in Table 4. Figure 9 presents typical synthetic $\mathrm{SN} /$ host spectra, with relative amplitudes determined from the PCA analysis.

\section{CONCLUSION AND FUTURE WORK}

We have presented the follow-up spectroscopy for the fall 2005 season of the SDSS-II SN Survey. A semi-quantitative procedure of SN identification is developed, based on crosscorrelation techniques. The rvsao.xcsao $r$ value and the overlap of template and data in wavelength space are useful guides to the quality of the fit. Nevertheless, a fair amount of human judgment is required to flag solutions associated with false local minima for low-S/N spectra. Using this procedure, we have determined accurate redshifts from both SN spectra and host spectra within $\delta z_{\mathrm{SN}} \approx 0.005$ and $\delta z_{\mathrm{gal}} \approx 0.0005$, respectively. The typical uncertainty in phase is $\sim 3 \mathrm{~d}$. 
Table 4

SDSS-II SNe Spectral Decomposition and Host Analysis Results

\begin{tabular}{|c|c|c|c|c|c|c|c|c|}
\hline SNID $^{\mathrm{a}}$ & $\mathrm{Tel}^{\mathrm{b}}$ & Epoch $_{p}{ }^{c}$ & $\mathrm{SN}_{\mathrm{s}}{ }^{\mathrm{d}}$ & Epoch $_{\text {sh }}{ }^{\mathrm{e}}$ & Host $_{\mathrm{sh}}{ }^{\mathrm{f}}$ & SN Frac ${ }^{g}$ & $\chi_{v}^{2 \mathrm{~h}}$ & $Q^{\mathrm{i}}$ \\
\hline 1112 & HET & 10 & Ia/1999ac & 9 & $\mathrm{Sb}$ & $0.307 \pm 0.021$ & 1.88 & -1 \\
\hline 1253 & HET & 5 & Ia/1999ac & 7 & $\mathrm{Sb}$ & $0.669 \pm 0.019$ & 2.14 & 2.6 \\
\hline 2017 & HET & 3 & $\mathrm{Ia} / 1991 \mathrm{~T}$ & -5 & $\mathrm{Sbc} / \mathrm{Sc}$ & $0.351 \pm 0.018$ & 1.96 & -0.5 \\
\hline 2030 & HET & 29 & $\mathrm{Ia} / 1990 \mathrm{I}$ & 44 & $\mathrm{Sbc} / \mathrm{Sc}$ & $0.431 \pm 0.059$ & 4.93 & -0.1 \\
\hline 2533 & HET & 8 & $\mathrm{Ia} / 1991 \mathrm{~T}$ & 2 & $\mathrm{E} / \mathrm{S} 0$ & $0.584 \pm 0.03$ & 0.88 & 4.4 \\
\hline 3199 & HET & 8 & Ia/1997cy & 11 & $\mathrm{Sbc} / \mathrm{Sc}$ & $0.499 \pm 0.084$ & 2.12 & -0.6 \\
\hline 3377 & HET & 13 & $\mathrm{Ia} / 1999 \mathrm{ac}$ & 8 & $\mathrm{Sm} / \mathrm{Im}$ & $0.743 \pm 0.032$ & 2.54 & -0.2 \\
\hline 4000 & HET & 17 & $\mathrm{Ia} / 1997 \mathrm{br}$ & 14 & $\mathrm{Sbc} / \mathrm{Sc}$ & $0.395 \pm 0.033$ & 1.39 & 0 \\
\hline 4046 & HET & 10 & $\mathrm{Ia} / \mathrm{Ibc}$ & 15 & $\mathrm{E} / \mathrm{S} 0$ & $0.684 \pm 0.058$ & 2.62 & -6.3 \\
\hline 4241 & HET & 21 & Ia/1999aa & 14 & $\mathrm{Sm} / \mathrm{Im}$ & $0.66 \pm 0.08$ & 1.12 & 0.1 \\
\hline 4577 & HET & 9 & Ia?/1991T & 7 & $\mathrm{Sb}$ & $0.346 \pm 0.036$ & 1.45 & 0.3 \\
\hline 4679 & HET & 2 & $\mathrm{Ia} / 1991 \mathrm{~T}$ & 2 & $\mathrm{Sbc} / \mathrm{Sc}$ & $0.605 \pm 0.032$ & 1.17 & 3 \\
\hline 5183 & HET & 13 & Ia/1999aa & 2 & $\mathrm{Sb}$ & $0.471 \pm 0.031$ & 0.69 & 1.3 \\
\hline 5588 & HET & 10 & $\mathrm{Ia} / \mathrm{Ibc}$ & 8 & E/S0 & $0.321 \pm 0.043$ & 0.37 & 0.3 \\
\hline 5736 & HET & 17 & $\mathrm{Ia} / 2000 \mathrm{cx}$ & 14 & Unknown & $0.984 \pm 0.034$ & 1.73 & 1.5 \\
\hline 5966 & HET & 19 & $\mathrm{Ia} / 1997 \mathrm{br}$ & 19 & $\mathrm{Sbc} / \mathrm{Sc}$ & $0.592 \pm 0.061$ & 0.59 & -0.4 \\
\hline 6100 & HET & 21 & $\mathrm{Ia} / 1997 \mathrm{br}$ & 10 & $\mathrm{Sbc} / \mathrm{Sc}$ & $0.467 \pm 0.032$ & 0.86 & -0.8 \\
\hline 6127 & HET & 9 & $\mathrm{Ia} / \mathrm{Ibc}$ & -1 & $\mathrm{Sb}$ & $0.313 \pm 0.04$ & 1.2 & 1.1 \\
\hline 6192 & HET & 10 & Ia/1999ac & 8 & $\mathrm{E} / \mathrm{S} 0$ & $0.768 \pm 0.035$ & 1.25 & -0.7 \\
\hline 6649 & HET & 4 & $\mathrm{Ia} / 1999 \mathrm{ac}$ & 2 & Unknown & $0.991 \pm 0.03$ & 1.09 & 13.9 \\
\hline 6777 & HET & 17 & $\mathrm{Ia} / 1997 \mathrm{br}$ & 7 & $\mathrm{Sm} / \mathrm{Im}$ & $0.55 \pm 0.068$ & 0.43 & -0.4 \\
\hline 6777 & HET & 20 & Ia/1999ac & 6 & Unknown & $0.9 \pm 0.097$ & 0.53 & -0.9 \\
\hline 6780 & HET & 12 & $\mathrm{Ia} / 1991 \mathrm{~T}$ & 9 & Unknown & $0.847 \pm 0.071$ & 2.32 & -1.7 \\
\hline 6924 & HET & 5 & Ia/1999ac & 6 & Unknown & $0.954 \pm 0.027$ & 0.88 & 8.7 \\
\hline 7143 & HET & 1 & Ia/1999aa & 6 & E/S0 & $0.576 \pm 0.029$ & 1.14 & 3.8 \\
\hline 7475 & HET & 5 & $\mathrm{Ia} / 1991 \mathrm{~T}$ & 2 & $\mathrm{Sm} / \mathrm{Im}$ & $0.881 \pm 0.034$ & 2.46 & 1.8 \\
\hline 7779 & HET & 8 & $\mathrm{Ia} / 1999 \mathrm{ac}$ & 4 & E/S0 & $0.881 \pm 0.052$ & 1.08 & 2.8 \\
\hline 8030 & HET & 8 & $\mathrm{Ia} / 1999 \mathrm{ac}$ & 6 & $\mathrm{Sa}$ & $0.893 \pm 0.069$ & 1.09 & 1.9 \\
\hline 8046 & HET & -2 & $\mathrm{Ia} / \mathrm{Ibc}$ & -10 & $\mathrm{E} / \mathrm{S} 0$ & $0.33 \pm 0.012$ & 1.6 & 0.2 \\
\hline 8213 & HET & 13 & $\mathrm{Ia} / 1997 \mathrm{br}$ & 20 & E/S0 & $0.4 \pm 0.016$ & 2.75 & -1 \\
\hline 8495 & HET & 23 & $\mathrm{Ia} / 1991 \mathrm{~T}$ & 15 & $\mathrm{Sb}$ & $0.276 \pm 0.023$ & 3.17 & -0.4 \\
\hline 8598 & HET & 5 & $\mathrm{Ia} / 1991 \mathrm{~T}$ & 9 & $\mathrm{Sm} / \mathrm{Im}$ & $0.696 \pm 0.071$ & 1.36 & 0.3 \\
\hline 9045 & HET & 15 & $\mathrm{Ia} / 1991 \mathrm{~T}$ & 9 & $\mathrm{Sm} / \mathrm{Im}$ & $0.549 \pm 0.032$ & 0.65 & 1.3 \\
\hline 9457 & HET & 11 & $\mathrm{Ia} / 1993 \mathrm{~J}$ & -5 & $\mathrm{Sb}$ & $0.351 \pm 0.039$ & 1.35 & 0.1 \\
\hline 9467 & HET & 18 & Ia/1999aa & 16 & $\mathrm{Sb}$ & $0.338 \pm 0.027$ & 4.07 & 0.5 \\
\hline 11206 & HET & 19 & $\mathrm{Ia} / 1998 \mathrm{bw}$ & 12 & $\mathrm{Sb}$ & $0.373 \pm 0.022$ & 0.17 & 1.2 \\
\hline 11320 & HET & 22 & Ia/1997cy & 12 & $\mathrm{Sbc} / \mathrm{Sc}$ & $0.447 \pm 0.103$ & 1.2 & -0.6 \\
\hline 11452 & HET & 16 & $\mathrm{Ia} / 1991 \mathrm{~T}$ & 11 & $\mathrm{Sm} / \mathrm{Im}$ & $0.827 \pm 0.053$ & 1.18 & 0.1 \\
\hline 11557 & HET & -1 & Ia/1999aа & 6 & $\mathrm{Sb}$ & $0.765 \pm 0.047$ & 0.63 & 2.1 \\
\hline 11864 & HET & 16 & $\mathrm{Ia} / 1991 \mathrm{~T}$ & 9 & $\mathrm{Sbc} / \mathrm{Sc}$ & $0.577 \pm 0.047$ & 0.94 & -1.1 \\
\hline 12136 & HET & 7 & Ia/1999ac & 12 & Unknown & $0.983 \pm 0.038$ & 0.67 & 1.5 \\
\hline 1166 & Subaru & 17 & $\mathrm{Ia} / 1997 \mathrm{br}$ & 8 & $\mathrm{Sa}$ & $0.607 \pm 0.023$ & 1.03 & -3.1 \\
\hline 1688 & Subaru & 14 & $\mathrm{Ia} / 1999 \mathrm{ac}$ & 9 & $\mathrm{Sb}$ & $0.464 \pm 0.015$ & 1.36 & -1.1 \\
\hline 2165 & Subaru & 7 & $\mathrm{Ia} / 1999 \mathrm{ac}$ & 7 & $\mathrm{E} / \mathrm{S} 0$ & $0.928 \pm 0.005$ & 1.47 & 0.7 \\
\hline 2330 & Subaru & 8 & $\mathrm{Ia} / 1999 \mathrm{ac}$ & 8 & $\mathrm{E} / \mathrm{S} 0$ & $0.702 \pm 0.005$ & 1.85 & 6.5 \\
\hline 2422 & Subaru & 4 & $\mathrm{Ia} / 1999 \mathrm{ac}$ & 1 & $\mathrm{Sbc} / \mathrm{Sc}$ & $0.727 \pm 0.01$ & 2.21 & 17.5 \\
\hline 2635 & Subaru & 31 & Ia/1999aa & 29 & $\mathrm{E} / \mathrm{S} 0$ & $0.837 \pm 0.011$ & 1.7 & -3.2 \\
\hline 2789 & Subaru & 10 & Ia/1999ac & 5 & $\mathrm{E} / \mathrm{S} 0$ & $0.938 \pm 0.009$ & 0.91 & 3.8 \\
\hline 2992 & Subaru & 30 & Ia/1999aа & 30 & $\mathrm{E} / \mathrm{S} 0$ & $0.484 \pm 0.013$ & 1.71 & -2.8 \\
\hline 3080 & Subaru & 24 & Ia/1999aa & 16 & $\mathrm{E} / \mathrm{S} 0$ & $0.505 \pm 0.007$ & 2.95 & -18.7 \\
\hline 3451 & Subaru & -2 & $\mathrm{Ia} / 1991 \mathrm{~T}$ & 1 & $\mathrm{Sb}$ & $0.595 \pm 0.011$ & 1.29 & 6.3 \\
\hline 3452 & Subaru & -6 & $\mathrm{Ia} / 1991 \mathrm{~T}$ & -5 & $\mathrm{Sbc} / \mathrm{Sc}$ & $0.617 \pm 0.011$ & 1.22 & 6.8 \\
\hline 5391 & Subaru & 13 & $\mathrm{Ia} / 1997 \mathrm{br}$ & 13 & E/S0 & $0.906 \pm 0.011$ & 1.09 & -1.2 \\
\hline 5533 & Subaru & 6 & Ia/1999ac & 7 & $\mathrm{E} / \mathrm{S} 0$ & $0.887 \pm 0.004$ & 2.23 & -1.2 \\
\hline 5717 & Subaru & 9 & Ia/1999ac & 5 & $\mathrm{Sb}$ & $0.67 \pm 0.012$ & 3.76 & 7 \\
\hline 5737 & Subaru & 9 & Ia/1999ac & 7 & $\mathrm{Sb}$ & $0.599 \pm 0.014$ & 1.32 & 3.2 \\
\hline 5751 & Subaru & 36 & $\mathrm{Ia} / 1999 \mathrm{ac}$ & 40 & $\mathrm{E} / \mathrm{S} 0$ & $0.229 \pm 0.009$ & 10.84 & -3.2 \\
\hline 5844 & Subaru & 15 & Ia/1999ac & 6 & $\mathrm{E} / \mathrm{S} 0$ & $0.873 \pm 0.013$ & 1.54 & 8.3 \\
\hline 5944 & Subaru & 34 & Ia/1999ac & 31 & $\mathrm{E} / \mathrm{S} 0$ & $0.852 \pm 0.008$ & 3.48 & -14 \\
\hline 5957 & Subaru & 6 & $\mathrm{Ia} / 1999 \mathrm{ac}$ & 6 & $\mathrm{E} / \mathrm{S} 0$ & $0.925 \pm 0.009$ & 1.03 & -1.2 \\
\hline 6057 & Subaru & 37 & $\mathrm{Ia} / 1999 \mathrm{ac}$ & 30 & $\mathrm{Sm} / \mathrm{Im}$ & $0.465 \pm 0.013$ & 37.92 & -2.8 \\
\hline 6108 & Subaru & 0 & Ia/1999ac & 3 & $\mathrm{Sa}$ & $0.681 \pm 0.011$ & 1.65 & 6.5 \\
\hline 6196 & Subaru & 7 & Ia/1999ac & 0 & $\mathrm{E} / \mathrm{S} 0$ & $0.364 \pm 0.006$ & 1.65 & 13.4 \\
\hline 6249 & Subaru & 0 & $\mathrm{Ia} / \mathrm{Ibc}$ & -1 & $\mathrm{Sbc} / \mathrm{Sc}$ & $0.613 \pm 0.009$ & 1.63 & 25.6 \\
\hline
\end{tabular}


Table 4

(Continued)

\begin{tabular}{|c|c|c|c|c|c|c|c|c|}
\hline SNID $^{\mathrm{a}}$ & $\mathrm{Tel}^{\mathrm{b}}$ & $\operatorname{Epoch}_{p}{ }^{\mathrm{c}}$ & $\mathrm{SN}_{s}{ }^{\mathrm{d}}$ & $\operatorname{Epoch}_{s h}{ }^{\mathrm{e}}$ & Host $_{s h}{ }^{\mathrm{f}}$ & SN Frac ${ }^{g}$ & $\chi_{\nu}^{2 \mathrm{~h}}$ & $Q^{\mathrm{i}}$ \\
\hline 6406 & Subaru & 27 & $\mathrm{Ia} / 1999 \mathrm{aa}$ & 30 & E/S0 & $0.484 \pm 0.005$ & 5.55 & 7.1 \\
\hline 6699 & Subaru & 7 & $\mathrm{Ia} / 1999 \mathrm{ac}$ & 2 & E/S0 & $0.771 \pm 0.014$ & 0.84 & 13.9 \\
\hline 9032 & Subaru & 14 & $\mathrm{Ia} / 1999 \mathrm{ac}$ & 13 & $\mathrm{E} / \mathrm{S} 0$ & $0.918 \pm 0.005$ & 1.56 & 10.9 \\
\hline 9207 & Subaru & 15 & $\mathrm{Ia} / 1999 \mathrm{ac}$ & 9 & E/S0 & $0.612 \pm 0.018$ & 1.24 & -2.1 \\
\hline 10449 & Subaru & 6 & $\mathrm{Ia} / 1999 \mathrm{ac}$ & 6 & $\mathrm{Sa}$ & $0.748 \pm 0.015$ & 1.44 & 7.1 \\
\hline 10550 & Subaru & 13 & $\mathrm{Ia} / 1999 \mathrm{ac}$ & 9 & E/S0 & $0.797 \pm 0.016$ & 1.28 & 3.4 \\
\hline 2053 & HET & 20 & Ic(02ap)/1997br & 3 & E/S0 & $0.732 \pm 0.038$ & 0.81 & 15 \\
\hline 2366 & HET & 58 & $\mathrm{II}(99 \mathrm{em}) / 2 \mathrm{n}$ & 38 & $\mathrm{Sb}$ & $0.137 \pm 0.03$ & 0.57 & 30.6 \\
\hline 2943 & HET & 13 & $\mathrm{Ia} / 1997 \mathrm{br}$ & 14 & $\mathrm{Sbc} / \mathrm{Sc}$ & $0.831 \pm 0.067$ & 1.28 & -1.3 \\
\hline 5350 & HET & 8 & $\mathrm{Ia} / 1997 \mathrm{br}$ & -5 & $\mathrm{Sa}$ & $0.268 \pm 0.013$ & 3.11 & -1.4 \\
\hline 5821 & HET & 18 & $\mathrm{Ia} ? / \mathrm{Ibc}$ & -5 & E/S0 & $0.292 \pm 0.033$ & 0.63 & -0.6 \\
\hline 5821 & HET & 16 & Ia?/1991T & 8 & $\mathrm{Sbc} / \mathrm{Sc}$ & $0.321 \pm 0.07$ & 0.63 & -0.6 \\
\hline 6137 & HET & 11 & $\mathrm{Ia} / 1999 \mathrm{ac}$ & 14 & $\mathrm{Sbc} / \mathrm{Sc}$ & $0.461 \pm 0.034$ & 0.94 & 0.7 \\
\hline 6315 & HET & 1 & Ia /Ibc & -4 & $\mathrm{Sbc} / \mathrm{Sc}$ & $0.557 \pm 0.018$ & 2.07 & 10.4 \\
\hline 6696 & HET & 5 & Ia?/1998bw & -5 & $\mathrm{Sbc} / \mathrm{Sc}$ & $0.304 \pm 0.03$ & 1.96 & -4.5 \\
\hline 6852 & HET & -3 & Ia?/1991T & 1 & $\mathrm{Sbc} / \mathrm{Sc}$ & $0.194 \pm 0.021$ & 1.12 & -2.7 \\
\hline 7335 & HET & 8 & Ia?/1991T & 45 & Unknown & $0.813 \pm 0.133$ & 1.63 & -1.3 \\
\hline 7426 & HET & -7 & Ia?/1991T & 2 & $\mathrm{Sbc} / \mathrm{Sc}$ & $0.441 \pm 0.101$ & 0.95 & -1.5 \\
\hline 7460 & HET & 7 & Ia?/IIl & -5 & E/S0 & $0.353 \pm 0.03$ & 2.67 & 0.1 \\
\hline 7947 & HET & 7 & Ia?/1998bw & 2 & $\mathrm{Sbc} / \mathrm{Sc}$ & $0.475 \pm 0.029$ & 1.26 & 0 \\
\hline 8707 & HET & 28 & Ia?/IIl & 15 & $\mathrm{Sm} / \mathrm{Im}$ & $0.518 \pm 0.103$ & 0.46 & -0.6 \\
\hline 9118 & HET & 7 & Ic(02ap)/hyper & -4 & $\mathrm{Sbc} / \mathrm{Sc}$ & $0.413 \pm 0.024$ & 4.85 & 4.4 \\
\hline 9273 & HET & 8 & Ic(02ap)/hyper & -5 & $\mathrm{Sb}$ & $0.122 \pm 0.017$ & 7.34 & -0.1 \\
\hline 10106 & HET & 39 & Ia?/1991T & 30 & $\mathrm{Sa}$ & $0.147 \pm 0.02$ & 0.95 & -1.3 \\
\hline 10297 & HET & 16 & II $(99 \mathrm{em}) / 1998 \mathrm{bw}$ & 38 & $\mathrm{Sbc} / \mathrm{Sc}$ & $0.057 \pm 0.018$ & 0.98 & 23.1 \\
\hline 11332 & HET & 2 & Ibc/hyper & 12 & $\mathrm{Sa}$ & $0.17 \pm 0.017$ & 8.96 & -2.7 \\
\hline 11332 & HET & 8 & Ibc/hyper & 22 & E/S0 & $0.323 \pm 0.015$ & 12.89 & -0.4 \\
\hline 11332 & HET & 64 & Ibc/IIn & 1 & $\mathrm{Sa}$ & $0.113 \pm 0.012$ & 6.37 & -0.2 \\
\hline 11650 & HET & -1 & $\mathrm{Ia} / 1999 \mathrm{ac}$ & 13 & Unknown & $0.938 \pm 0.033$ & 1.21 & -1.2 \\
\hline 1686 & Subaru & 11 & Ia?/1991T & 28 & E/S0 & $0.617 \pm 0.016$ & 0.95 & -1.3 \\
\hline 2661 & Subaru & 25 & II(99em)/IIl & 6 & $\mathrm{Sa}$ & $0.205 \pm 0.007$ & 1.07 & 1.8 \\
\hline 6471 & Subaru & 3 & $\mathrm{II}(99 \mathrm{em}) / \mathrm{Ibc}$ & 5 & $\mathrm{Sbc} / \mathrm{Sc}$ & $0.413 \pm 0.012$ & 1.07 & -0.2 \\
\hline
\end{tabular}

Notes.

a SDSS internal SN ID.

${ }^{\mathrm{b}}$ Spectroscopic follow-up telescope.

c Observation date of the SN relative to $B$-band maximum brightness estimated from light-curve fits.

d The best and second-best SN type from cross-correlation analysis. "Ia?" indicates best fit to an SN Ia template, but a reasonable fit to the next-best type.

${ }^{\text {e }}$ Age of the $\mathrm{SN}$ relative to $B$-band maximum brightness estimated from spectrum decomposition.

${ }^{\mathrm{f}}$ Host-galaxy type ID from spectral decomposition. Unknown indicates too little host light for type ID.

$\mathrm{g}$ Estimate of the SN light fraction in the observed spectrum, with statistical error (the maximum value of the asymmetric errors obtained from the PCA code).

${ }^{\mathrm{h}}$ Reduced $\chi^{2}$ value.

${ }^{\mathrm{i}}$ Quality factor $Q=\frac{\Delta \chi_{v}^{2} \sqrt{v}}{\chi_{v}^{2}}$, which estimates the significance of the $\chi^{2}$ differentiation between the best two SN types. Negative values indicate a selected type differing from that determined by the cross-correlation analysis.

j SNe above the line have extinction estimates from MCLS2k2 analysis (Jha et al. 2007). Those below the line have $A_{V}$ from light-curve fitting using the frame difference photometry.

We have also described our efforts to quantify host-galaxy contamination using a combined $\chi^{2}$ fitting and PCA analysis, which provides an efficient way to give useful decompositions into SN and host-galaxy spectra with $<10 \%$ accuracy, given known SN types from cross-correlation analysis and host-galaxy extinction estimated from the multi-band light curves. Typically, when the spectrum contains more than $60 \%$ host-galaxy light, the host type is well constrained. Both spectroscopic analyses show good agreement in estimating the photometric epoch.

Using our quantitative measurement of the SN light fraction, we can make galaxy-subtracted SN spectra with estimates of the residual galaxy contamination. With a $>50 \%$ larger yield from the second season, 2006, and an anticipated large number of $\mathrm{SNe}$ in the third and final seasons, the classified and hostsubtracted spectra will provide a large and uniform data set with which to study SN spectral variations and evolution. We expect that this will help substantially in calibrating our use of $\mathrm{SNe}$ as distance indicators and will allow improved calibration of the SDSS-II and future larger (e.g., the Panoramic Survey Telescope and Rapid Response System, the Dark Energy Survey, the Large Survey Telescope, and the Joint Dark Energy Mission) SN samples. 

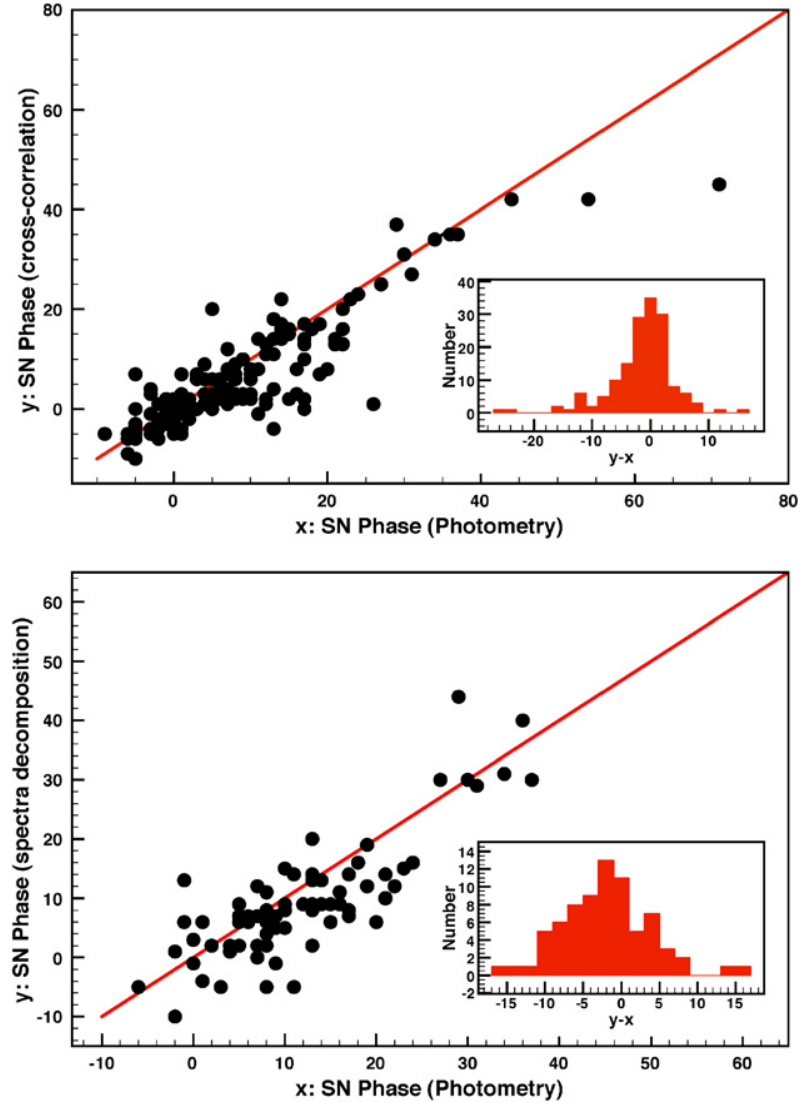

Figure 8. Two panels comparing different-epoch measurements of confirmed $\mathrm{SNe}$ Ia. The upper panel shows the SN phase (days past peak $B$-band magnitude) measured from cross-correlation analysis of the spectra against the photometric phase. The lower plot shows the SN phase measured from the PCA + templatefitting program against the photometric phase. The insets show histograms of the scatter between the two epoch measurements, with $y$ being the phase determined from either cross-correlation analysis or the spectra decomposition analysis and $x$ being the phase determined from light-curve fitting.

(A color version of this figure is available in the online journal)

Funding for the SDSS and SDSS-II has been provided by the Alfred P. Sloan Foundation, the Participating Institutions, the National Science Foundation (NSF), the U.S. Department of Energy, the National Aeronautics and Space Administration (NASA), the Japanese Monbukagakusho, the Max Planck Society, and the Higher Education Funding Council for England. The SDSS Web Site is http://www.sdss.org/.

The SDSS is managed by the ARC for the Participating Institutions. The Participating Institutions are the American Museum of Natural History, Astrophysical Institute Potsdam, University of Basel, University of Cambridge, Case Western Reserve University, University of Chicago, Drexel University, Fermilab, the Institute for Advanced Study, the Japan Participation Group, Johns Hopkins University, the Joint Institute for Nuclear Astrophysics, the Kavli Institute for Particle Astrophysics and Cosmology, the Korean Scientist Group, the Chinese Academy of Sciences (LAMOST), Los Alamos National Laboratory, the Max Planck Institute for Astronomy (MPIA), the Max Planck Institute for Astrophysics (MPA), New Mexico State University, Ohio State University, University of Pittsburgh, University of Portsmouth, Princeton University, the United States Naval Observatory, and the University of Washington.
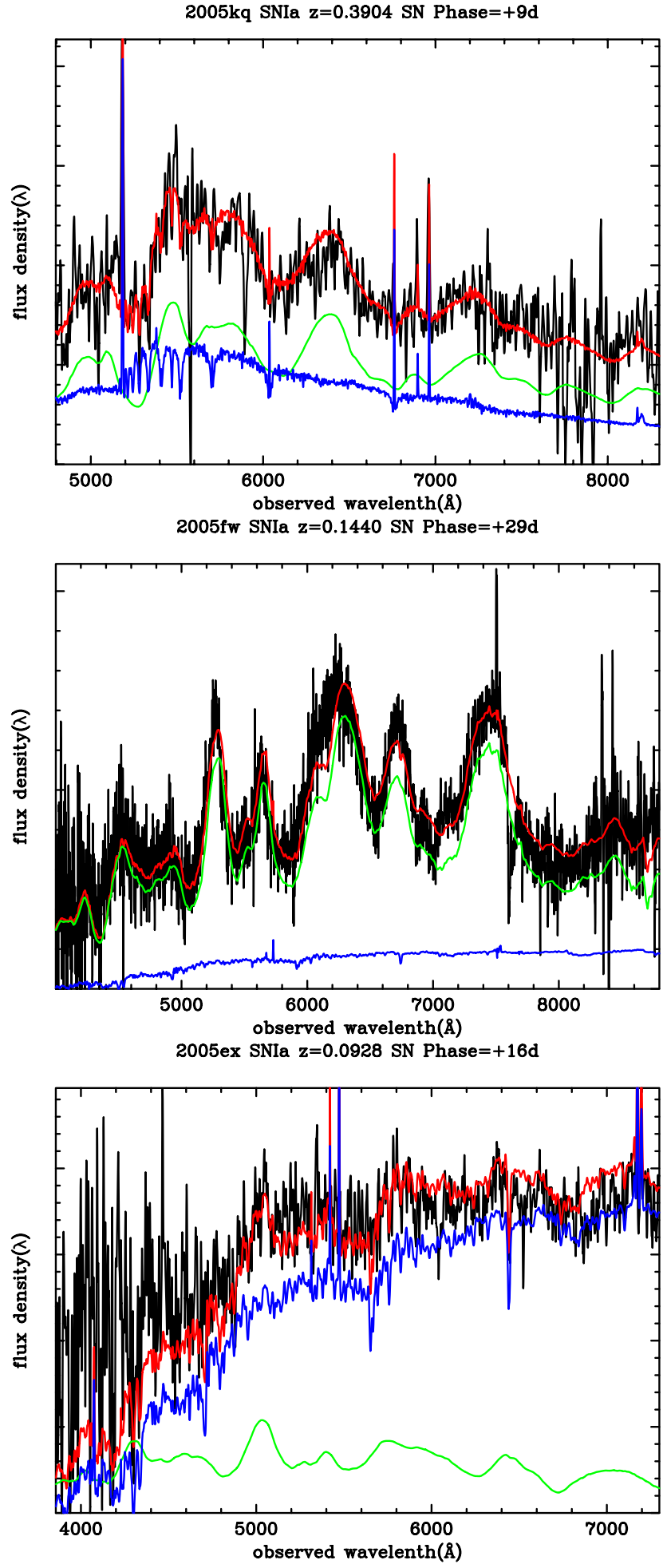

Figure 9. Examples of PCA spectral decomposition. The data (black) are overplotted with the best-fitted combined model (red), with host-galaxy (blue) and SN (green) components. The header of each figure shows the name, redshift, and phase of the SN. The wavelength is shown in the observed frame. The HET spectrum (upper left, resolution: $20 \AA$ ) has almost an equal amount of SN and host light. The Subaru spectrum (middle left, resolution: 8-12 $\AA$ ) has little host contamination, while the MDM spectrum (bottom left, resolution: $15 \AA$ ) is dominated by the host.

(A color version of this figure is available in the online journal) 
This work is based in part on observations made at the following telescopes. The HET is a joint project of the University of Texas at Austin, the Pennsylvania State University, Stanford University, Ludwig-Maximillians-Universität München, and Georg-August-Universität Göttingen. The HET is named in honor of its principal benefactors, William P. Hobby and Robert E. Eberly. The Marcario Low-Resolution Spectrograph is named for Mike Marcario of High Lonesome Optics, who fabricated several optical elements for the instrument but died before its completion; it is a joint project of the HST partnership and the Instituto de Astronom de la Universidad Nacional Autonoma de Mexico. We thank the HET resident astronomers (John Caldwell, Heinz Edelmann, Steve Odewahn, and Matthew Shetrone) for their continued effort and support with the HET observations. The APO $3.5 \mathrm{~m}$ telescope is owned and operated by the ARC. We thank the observatory director, Suzanne Hawley, and site manager, Bruce Gillespie, for their support of this project. The Subaru Telescope is operated by the National Astronomical Observatory of Japan. The William Herschel Telescope is operated by the Isaac Newton Group, and the Nordic Optical Telescope is operated jointly by Denmark, Finland, Iceland, Norway, and Sweden, both on the island of La Palma in the Spanish Observatorio del Roque de los Muchachos of the Instituto de Astrofisica de Canarias. Observations at the ESO New Technology Telescope at La Silla Observatory were made under programme IDs 77.A-0437, 78.A-0325, and 79.A-0715. Kitt Peak National Observatory, National Optical Astronomy Observatories (NOAO), is operated by the Association of Universities for Research in Astronomy, Inc. (AURA) under cooperative agreement with the NSF. The WIYN Observatory is a joint facility of the University of Wisconsin-Madison, Indiana University, Yale University, and NOAO. The W. M. Keck Observatory is operated as a scientific partnership among the California Institute of Technology, the University of California, and NASA. The Observatory was made possible by the generous financial support of the W. M. Keck Foundation. The South African Large Telescope of the South African Astronomical Observatory is operated by a partnership between the National Research Foundation of South Africa, Nicolaus Copernicus Astronomical Center of the Polish Academy of Sciences, the HobbyEberly Telescope Board, Rutgers University, Georg-AugustUniversität Göttingen, University of Wisconsin-Madison, University of Canterbury, University of North Carolina-Chapel Hill, Dartmouth College, Carnegie Mellon University, and the United Kingdom SALT consortium. A.V.F.'s supernova group at U.C. Berkeley is supported by NSF grant AST-0607485.

This work is also supported in part by the U.S. Department of Energy under contract number DE-AC0276SF00515.

\section{REFERENCES}

Adelman-McCarthy, J., et al. 2007, ApJS, 172, 634

Aldering, G., et al. 2006, ApJ, 650, 510

Altavilla, G., et al. 2004, MNRAS, 349, 1344

Astier, P., et al. 2006, A\&A, 447, 31

Balland, C., et al. 2006, A\&A, 445, 387

Benetti, S., et al. 2005, ApJ, 623, 1011

Blondin, S., \& Tonry, J. 2007a, AIPC, 924, 312

Blondin, S., \& Tonry, J. L. 2007b, ApJ, 666, 1024

Blondin, S., Walsh, J. R., Leibundgut, B., \& Sainton, G. 2005, A\&A, 431, 757

Branch, D., Fisher, A., \& Nugent, P. 1993, AJ, 106, 2383

Branch, D., \& Tammann, G. A. 1992, ARA\&A, 30, 359

Branch, D., et al. 2002, ApJ, 566, 1005

Cardeli, J. A., Calyton, G. C., \& Mathis, J. S. 1989, ApJ, 345, 245

Chornock, R., Filippenko, A. V., Branch, D., Foley, R. J., Jha, S., \& Li, W. 2006, PASP, 118, 722
Clocchiatti, A., et al. 1996, ApJ, 462, 462

Connolly, A. J, Szalay, A. S., Bershady, M. A., Kinney, A. L., \& Calzetti, D. 1995, AJ, 110, 1071

Copin, Y., et al. 2006, New A Rev., 50, Issue 4-5, 436

Dilday, B., et al. 2008, ApJ, at press (arXiv:0801.3297)

Elmhamdi, A., Danziger, I. J., Cappellaro, E., Della Valle, M., Gouiffes, C., Phillips, M. M., \& Turatto, M. 2004, A\&A, 426, 963

Ferdinando, P., et al. 2001, ApJ, 555, 900

Filippenko, A. V. 1982, PASP, 94, 715

Filippenko, A. V. 1997, ARA\&A, 35, 309

Filippenko, A. V. 2005a, in The Fate of the Most Massive Stars, ed. R. Humphreys, \& K. Stanek (San Francisco, CA: ASP), 33

Filippenko, A. V. 2005b, in White Dwarfs: Cosmological and Galactic Probes, ed. E. M. Sion, S. Vennes, \& H. L. Shipman (Dordrecht: Springer), 97

Filippenko, A. V., Li, W. D., Treffers, P. R., \& Modjaz, M. 2001, PASP, 246, 121

Filippenko, A. V., et al. 1992a, ApJ, 384, L15

Filippenko, A. V., et al. 1992b, AJ, 104, 1543

Foley, R. J., et al. 2003, PASP, 115, 1220

Folkes, S., Lahav, O., \& Maddox, S. 1996, MNRAS, 283, 651

Frieman, J. A., et al. 2008, AJ, 135, 238

Fukugita, M., Ichikawa, T., Gunn, J. E., Doi, M., Shimasaku, K., \& Schneider, D. P. 1996, AJ, 111, 174

Gal-Yam, A., Ofek, E., \& Suemmer, O. 2002, MNRAS, 332, L73

Garavini, G., et al. 2004, AJ, 128, 387

Garnavich, P., et al. 2004, ApJ, 613, 1120

Gunn, J. E., et al. 1998, AJ, 116, 3040

Gunn, J. E., et al. 2006, AJ, 131, 2332

Hamuy, M., et al. 1996, AJ, 112, 2398

Hamuy, M., et al. 2002, AJ, 124, 417

Hamuy, M., et al. 2003, Nature, 424, 651

Hamuy, M., et al. 2006, PASP, 118, 2

Hill, G. J., et al. 1998, Proc. SPIE, 3355, 375

Hook, I., et al. 2005, AJ, 130, 2788

Howell, D. A., \& Wang, L. 2002, BAAS, 34, 1256

Howell, D. A., et al. 2005, ApJ, 634, 1190

Hsiao, E. Y., et al. 2007, ApJ, 663, 1187

Ivezic, Z., et al. 2007, AJ, 134, 973

Jha, S., Riess, A. G., \& Kirshner, R. P. 2007, ApJ, 659, 122

Jha, S., et al. 1999, ApJS, 125, 73

Jha, S., et al. 2006, AJ, 131, 527

Knop, R. A., et al. 2003, ApJ, 598, 102

Krisciunas, K., et al. 2006, AJ, 131, 1639

Leonard, D. C., et al. 2002, PASP, 114, 35

Li, W. D., et al. 2000, in Cosmic Explosions, ed. S. S. Holt, \& W. W. Zhang (New York: AIP), 103

Lidman, C., et al. 2005, A\&A, 430, 843

Madgwick, D. S., Hewett, P. C., Mortlock, D. J., \& Wang, L. 2003, ApJ, 599, L33

Madgwick, D. S., et al. 2002, MNRAS, 333, 133

Matheson, T., et al. 2001, ApJ, 121, 1648

Matheson, T., et al. 2003, ApJ, 599, 394

Matheson, T., et al. 2005, AJ, 129, 2352

Miknaitis, G., et al. 2007, ApJ, 666, 674

Nugent, P., Kim, A., \& Perlmutter, S. 2002, PASP, 114, 803

Oke, J. B., et al. 1995, PASP, 107, 375

Perlmutter, S., et al. 1999, ApJ, 517, 565

Phillips, M. M. 1993, ApJ, 413, L105

Phillips, M. M., et al. 2007, PASP, 119, 360

Prieto, J. L., et al. 2007, AJ, submitted (arXiv:0706.4088)

Pun, C. S. J., et al. 1995, ApJ, 99, 223

Quimby, R., Höflich, P., \& Wheeler, J. C. 2007, ApJ, 666, 1083

Riess, A. G. 1996, Ph.D. thesis, Harvard Univ.

Riess, A. G., Press, W. H., \& Kirshner, R. P. 1995, ApJ, 438, L17

Riess, A. G., et al. 1998, AJ, 116, 1009

Riess, A. G., et al. 1999, AJ, 117, 707

Riess, A. G., et al. 2004, AJ, 607, 665

Sako, M., et al. 2008, AJ, 135, 348

Shetrone, M., et al. 2007, PASP, 119, 556

Stanek, K. Z., et al. 2003, ApJ, 591, 17

Stathakis, R., et al. 2000, MNRAS, 314, 807

Tonry, J., \& Davis, M. 1979, AJ, 84, 10

Turatto, M., Benetti, S., Cappellaro, E., Danziger, I. J., Della Valle, M., Gouiffes, C., Mazzali, P. A., \& Patat, F. 1996, MNRAS, 283, 1

Wood-Vasey, W. M., et al. 2007, ApJ, 666, 694

Yip, C. W., et al. 2004, AJ, 128, 585

York, D. G., et al. 2000, AJ, 120, 1579 